\title{
Multisymplectic Lagrangian and Hamiltonian Formalisms of Classical Field Theories
}

\author{
Narciso ROMÁN-ROY \\ Dept. Matemática Aplicada IV, Edificio C-3, Campus Norte UPC, \\ C/ Jordi Girona 1, E-08034 Barcelona, Spain \\ E-mail: nrr@ma4.upc.edu \\ URL: http://www-ma4.upc.edu/ nrr/
}

Received July 02, 2009, in final form October 30, 2009; Published online November 06, 2009

doi:10.3842/SIGMA.2009.100

\begin{abstract}
This review paper is devoted to presenting the standard multisymplectic formulation for describing geometrically classical field theories, both the regular and singular cases. First, the main features of the Lagrangian formalism are revisited and, second, the Hamiltonian formalism is constructed using Hamiltonian sections. In both cases, the variational principles leading to the Euler-Lagrange and the Hamilton-De Donder-Weyl equations, respectively, are stated, and these field equations are given in different but equivalent geometrical ways in each formalism. Finally, both are unified in a new formulation (which has been developed in the last years), following the original ideas of Rusk and Skinner for mechanical systems.
\end{abstract}

Key words: classical field theories; Lagrangian and Hamiltonian formalisms; fiber bundles; multisymplectic manifolds

2000 Mathematics Subject Classification: 70S05; 55R10; 53C80

\section{Introduction}

In recent years much work has been done with the aim of establishing the suitable geometrical structures for describing classical field theories.

There are different kinds of geometrical models for making a covariant description of classical field theories described by first-order Lagrangians. For instance, we have the so-called $k$-symplectic formalism which uses the $k$-symplectic forms introduced by Awane [4, 5, 6], and which coincides with the polysymplectic formalism described by Günther [46] (see also [84]). A natural extension of this is the $k$-cosymplectic formalism, which is the generalization to field theories of the cosymplectic description of non-autonomous mechanical systems [75, 76]. Furthermore, there are the polysymplectic formalisms developed by Sardanashvily et al. [39, 91] and Kanatchikov [52], which are based on the use of vector-valued forms on fiber bundles, and which are different descriptions of classical field theories than the polysymplectic one proposed by Günther. In addition, soldering forms on linear frame bundles are also polysymplectic forms, and their study and applications to field theory constitute the $k$-symplectic geometry developed by Norris [85, 86, 87]. There also exists the formalism based on using Lepagean forms, used for describing certain kinds of equivalent Lagrangian models with non-equivalent Hamiltonian descriptions [64, 65, 66, 67]. Finally, a new geometrical framework for field theories based on the use of Lie algebroids has been developed in recent works [72, 82, 83].

In this work, we consider only the multisymplectic models [18, 41, 43, 68, 79], first introduced by Tulczyjew and other authors [37, 40,60,61]. They arise from the study of multisymplectic manifolds and their properties (see [14, 15] for general references, and Appendix A.1 for a brief review); in particular, those concerning the behavior of multisymplectic Lagrangian and Hamiltonian systems. 
The usual way of working with field theories consists in stating their Lagrangian formalism $[3,11,17,26,27,37,39,40,92]$, and jet bundles are the appropriate domain for doing so. The construction of this formalism for regular and singular theories is reviewed in Section 2.

The Hamiltonian description presents different kinds of problems. For instance, the choice of the multimomentum bundle for developing the theory is not unique [29, 30], and different kinds of Hamiltonian systems can be defined, depending on this choice and on the way of introducing the physical content (the "Hamiltonian") [23, 25, 47, 48, 78, 88]. Here we present one of the most standard ways of defining Hamiltonian systems, which is based on using Hamiltonian sections [16]; although this construction can also be done taking Hamiltonian densities [16, 39, 79, 91]. In particular, the construction of Hamiltonian systems which are the Hamiltonian counterpart of Lagrangian systems is carried out by using the Legendre map associated with the Lagrangian system, and this problem has been studied by different authors in the (hyper) regular case [16, 92], and in the singular (almost-regular) case [39, 69, 91]. In Section 3 we review some of these constructions.

Another subject of interest in the geometrical description of classical field theories concerns the field equations. In the multisymplectic models, both in the Lagrangian and Hamiltonian formalisms, these equations can be derived from a suitable variational principle: the so-called Hamilton principle in the Lagrangian formalism and Hamilton-Jacobi principle in the Hamiltonian formulation $[3,23,26,30,37,40]$, and the field equations are usually written by using the multisymplectic form in order to characterize the critical sections which are solutions of the problem. In addition, these critical sections can be thought of as being the integral manifolds of certain kinds of integrable multivector fields or Ehresmann connections, defined in the bundles where the formalism is developed, and satisfying a suitable geometric equation which is the intrinsic formulation of the systems of partial differential equations locally describing the field [26, 27, 28, 69, 92]. All these aspects are discussed in Sections 2 and 3 (furthermore, a quick review on multivector fields and connections is given in Appendix A.2). Moreover, multivector fields are also used in order to state generalized Poisson brackets in the Hamiltonian formalism of field theories [34, 50, 51, 52, 88].

In ordinary mechanics there is also a unified formulation of Lagrangian and Hamiltonian formalisms [94], which is based on the use of the Whitney sum of the tangent and cotangent bundles (the velocity and momentum phase spaces of the system). This formalism has been generalized for non-autonomous mechanics [7, 20, 45] and recently for classical field theories [24, 71]. The main features of this formulation are explained in Section 4.

Finally, an example showing the application of these formalisms is analyzed in Section 5. A last section is devoted to make a discussion about the current status on the research on different topics concerning the multisymplectic approach to classical field theories.

We ought to point out that there are also geometric frameworks for describing the noncovariant or space-time formalism of field theories, where the use of Cauchy surfaces is the fundamental tool $[42,44,74]$. Nevertheless we do not consider these topics in this survey.

As a review paper, this work recovers results and contributions from several previous papers, such as [16, 24, 26, 27, 28, 30, 47, 69, 71, 88], among others.

In this paper, manifolds are real, paracompact, connected and $C^{\infty}$, maps are $C^{\infty}$, and sum over crossed repeated indices is understood.

\section{Lagrangian formalism}

\section{$2.1 \quad$ Lagrangian systems}

A classical field theory is described by the following elements: First, we have the configuration fibre bundle $\pi: E \rightarrow M$, with $\operatorname{dim} M=m$ and $\operatorname{dim} E=n+m$, where $M$ is an oriented 
manifold with volume form $\omega \in \Omega^{m}(M) . \pi^{1}: J^{1} \pi \rightarrow E$ is the first-order jet bundle of local sections of $\pi$, which is also a bundle over $M$ with projection $\bar{\pi}^{1}=\pi \circ \pi^{1}: J^{1} \pi \longrightarrow M$, and $\operatorname{dim} J^{1} \pi=n m+n+m$. We denote by $\left(x^{\nu}, y^{A}, v_{\nu}^{A}\right)(\nu=1, \ldots, m ; A=1, \ldots, n)$ natural coordinates in $J^{1} \pi$ adapted to the bundle structure and such that $\omega=\mathrm{d} x^{1} \wedge \cdots \wedge \mathrm{d} x^{m} \equiv \mathrm{d}^{m} x$. Second, we give the Lagrangian density, which is a $\bar{\pi}^{1}$-semibasic $m$-form on $J^{1} \pi$ and hence it can be expressed as $\mathcal{L}=£\left(\bar{\pi}^{1 *} \omega\right)$, where $£ \in \mathrm{C}^{\infty}\left(J^{1} \pi\right)$ is the Lagrangian function associated with $\mathcal{L}$ and $\omega$.

The bundle $J^{1} \pi$ is endowed with a canonical structure, $\mathcal{V} \in \Omega^{1}\left(J^{1} \pi\right) \otimes \Gamma\left(J^{1} \pi, \mathrm{V}\left(\pi^{1}\right)\right) \otimes$ $\Gamma\left(J^{1} \pi, \bar{\pi}^{1^{*}} \mathrm{~T} M\right)$, which is called the vertical endomorphism $[26,37,40,92]$ (here $\mathrm{V}\left(\pi^{1}\right)$ denotes the vertical subbundle with respect to the projection $\pi^{1}$, and $\Gamma\left(J^{1} \pi, \mathrm{V}\left(\pi^{1}\right)\right)$ the set of sections in the corresponding bundle). Then the Poincaré-Cartan $m$ and $(m+1)$-forms associated with $\mathcal{L}$ are defined as

$$
\Theta_{\mathcal{L}}:=i(\mathcal{V}) \mathcal{L}+\mathcal{L} \in \Omega^{m}\left(J^{1} \pi\right), \quad \Omega_{\mathcal{L}}:=-\mathrm{d} \Theta_{\mathcal{L}} \in \Omega^{m+1}\left(J^{1} \pi\right) .
$$

We have the following local expressions (where $\left.\mathrm{d}^{m-1} x_{\alpha} \equiv i\left(\frac{\partial}{\partial x^{\alpha}}\right) \mathrm{d}^{m} x\right)$ :

$$
\begin{aligned}
\Theta_{\mathcal{L}}= & \frac{\partial £}{\partial v_{\nu}^{A}} \mathrm{~d} y^{A} \wedge \mathrm{d}^{m-1} x_{\nu}-\left(\frac{\partial £}{\partial v_{\nu}^{A}} v_{\nu}^{A}-£\right) \mathrm{d}^{m} x \\
\Omega_{\mathcal{L}}= & -\frac{\partial^{2} £}{\partial v_{\nu}^{B} \partial v_{\alpha}^{A}} \mathrm{~d} v_{\nu}^{B} \wedge \mathrm{d} y^{A} \wedge \mathrm{d}^{m-1} x_{\alpha}-\frac{\partial^{2} £}{\partial y^{B} \partial v_{\alpha}^{A}} \mathrm{~d} y^{B} \wedge \mathrm{d} y^{A} \wedge \mathrm{d}^{m-1} x_{\alpha} \\
& +\frac{\partial^{2} £}{\partial v_{\nu}^{B} \partial v_{\alpha}^{A}} v_{\alpha}^{A} \mathrm{~d} v_{\nu}^{B} \wedge \mathrm{d}^{m} x+\left(\frac{\partial^{2} £}{\partial y^{B} \partial v_{\alpha}^{A}} v_{\alpha}^{A}-\frac{\partial £}{\partial y^{B}}+\frac{\partial^{2} £}{\partial x^{\alpha} \partial v_{\alpha}^{B}}\right) \mathrm{d} y^{B} \wedge \mathrm{d}^{m} x .
\end{aligned}
$$

Definition 1. $\left(J^{1} \pi, \Omega_{\mathcal{L}}\right)$ is said to be a Lagrangian system. The Lagrangian system and the Lagrangian function are said to be regular if $\Omega_{\mathcal{L}}$ is a multisymplectic $(m+1)$-form (i.e., 1-nondegenerate) $[16,26]$. Elsewhere they are singular (or non-regular).

The regularity condition is locally equivalent to $\operatorname{det}\left(\frac{\partial^{2} £}{\partial v_{\alpha}^{A} \partial v_{\nu}^{B}}(\bar{y})\right) \neq 0, \forall \bar{y} \in J^{1} \pi$. We must point out that, in field theories, the notion of regularity is not uniquely defined (for other approaches see, for instance, [9, 21, 22, 64, 66, 67]).

\subsection{Lagrangian field equations}

The Lagrangian field equations can be derived from a variational principle. In fact:

Definition 2. Let $\left(J^{1} \pi, \Omega_{\mathcal{L}}\right)$ be a Lagrangian system. Let $\Gamma(M, E)$ be the set of sections of $\pi$. Consider the map

$$
\begin{aligned}
\mathbf{L}: & \Gamma(M, E) \longrightarrow \mathbb{R}, \\
\phi & \mapsto \int_{M}\left(j^{1} \phi\right)^{*} \Theta_{\mathcal{L}},
\end{aligned}
$$

where the convergence of the integral is assumed. The variational problem for this Lagrangian system is the search of the critical (or stationary) sections of the functional $\mathbf{L}$, with respect to the variations of $\phi$ given by $\phi_{t}=\sigma_{t} \circ \phi$, where $\left\{\sigma_{t}\right\}$ is a local one-parameter group of any compact-supported $Z \in \mathfrak{X}^{\mathrm{V}(\pi)}(E)$ (the module of $\pi$-vertical vector fields in $E$ ), that is:

$$
\left.\frac{\mathrm{d}}{\mathrm{d} t}\right|_{t=0} \int_{M}\left(j^{1} \phi_{t}\right)^{*} \Theta_{\mathcal{L}}=0
$$

This is the Hamilton principle of the Lagrangian formalism. 
The Hamilton principle is equivalent to find a distribution $\mathcal{D}$ in $J^{1} \pi$ such that:

1. $\mathcal{D}$ is $m$-dimensional.

2. $\mathcal{D}$ is $\bar{\pi}^{1}$-transverse.

3. $\mathcal{D}$ is integrable (that is, involutive).

4. The integral manifolds of $\mathcal{D}$ are the canonical liftings to $J^{1} \pi$ of the critical sections of the Hamilton principle.

A distribution $\mathcal{D}$ satisfying 1 and 2 is associated with a connection in the bundle $\bar{\pi}^{1}: J^{1} \pi \rightarrow M$ (integrable if 3 holds), whose local expression is

$$
\nabla=\mathrm{d} x^{\mu} \otimes\left(\frac{\partial}{\partial x^{\nu}}+F_{\nu}^{A} \frac{\partial}{\partial y^{A}}+G_{\nu \rho}^{A} \frac{\partial}{\partial v_{\rho}^{A}}\right) .
$$

Furthermore, these kinds of integrable distributions and the corresponding connections are associated with classes of integrable (i.e., non-vanishing, locally decomposable and involutive) $\bar{\pi}^{1}$-transverse $m$-multivector fields in $J^{1} \pi$ (see Appendix A.2). If 2 holds, the local expression in natural coordinates of an element of one of these classes is

$$
\mathcal{X}=\bigwedge_{\nu=1}^{m} f\left(\frac{\partial}{\partial x^{\nu}}+F_{\nu}^{A} \frac{\partial}{\partial y^{A}}+G_{\nu \rho}^{A} \frac{\partial}{\partial v_{\rho}^{A}}\right), \quad\left(f \in \mathrm{C}^{\infty}\left(J^{1} \pi\right) \text { non-vanishing }\right)
$$

If, in addition, the integral sections are holonomic (that is, they are canonical liftings of sections of $\pi: E \rightarrow M)$, then the integrable connections and their associated classes of multivector fields are called holonomic. To be holonomic is equivalent to be integrable and semi-holonomic, that is, $F_{\nu}^{A}=v_{\nu}^{A}$ in the above local expressions. Then:

Theorem 1. Let $\left(J^{1} \pi, \Omega_{\mathcal{L}}\right)$ be a Lagrangian system. The following assertions on a section $\phi \in \Gamma(M, E)$ are equivalent:

1. $\phi$ is a critical section for the variational problem posed by the Hamilton principle.

2. $\left(j^{1} \phi\right)^{*} i(X) \Omega_{\mathcal{L}}=0, \forall X \in \mathfrak{X}\left(J^{1} \pi\right)$ (see $\left.[40]\right)$.

3. If $\left(U ; x^{\nu}, y^{A}, v_{\nu}^{A}\right)$ is a natural system of coordinates in $J^{1} \pi$, then $j^{1} \phi=\left(x^{\nu}, y^{A}\left(x^{\eta}\right), \frac{\partial y^{A}}{\partial x^{\nu}}\left(x^{\eta}\right)\right)$ in $U$ satisfies the Euler-Lagrange equations (see [26, 40])

$$
\frac{\partial £}{\partial y^{A}} \circ j^{1} \phi-\frac{\partial}{\partial x^{\mu}}\left(\frac{\partial £}{\partial v_{\mu}^{A}} \circ j^{1} \phi\right)=0 .
$$

4. $j^{1} \phi$ is an integral section of a class of holonomic multivector fields $\left\{\mathcal{X}_{\mathcal{L}}\right\} \subset \mathfrak{X}^{m}\left(J^{1} \pi\right)$ satisfying (see [27]):

$$
i\left(\mathcal{X}_{\mathcal{L}}\right) \Omega_{\mathcal{L}}=0, \quad \forall \mathcal{X}_{\mathcal{L}} \in\left\{\mathcal{X}_{\mathcal{L}}\right\}
$$

5. $j^{1} \phi$ is an integral section of a holonomic connection $\nabla_{\mathcal{L}}$ in $J^{1} \pi$ satisfying (see [69]):

$$
i\left(\nabla_{\mathcal{L}}\right) \Omega_{\mathcal{L}}=(m-1) \Omega_{\mathcal{L}}
$$

Proof. See [26, 27, 37, 40, 69, 92].

$(1 \Longleftrightarrow 2)$ Let $Z \in \mathfrak{X}^{\mathrm{V}(\pi)}(E)$ be a compact-supported vector field, and $V \subset M$ an open set such that $\partial V$ is a $(m-1)$-dimensional manifold and that $\bar{\tau}(\operatorname{supp}(Z)) \subset V$. We denote by 
$j^{1} Z \in \mathfrak{X}\left(J^{1} \pi\right)$ the canonical lifting of $Z$ to $J^{1} \pi$; and, if $Z \in \mathfrak{X}^{\mathrm{V}(\pi)}(E)$, then $j^{1} Z \in \mathfrak{X}^{\mathrm{V}\left(\bar{\pi}^{1}\right)}\left(J^{1} \pi\right)$ (see [26] for the details). Therefore

$$
\begin{aligned}
\left.\frac{d}{d t}\right|_{t=0} \int_{M}\left(j^{1} \phi_{t}\right)^{*} \Theta_{\mathcal{L}} & =\left.\frac{d}{d t}\right|_{t=0} \int_{V}\left(j^{1} \phi_{t}\right)^{*} \Theta_{\mathcal{L}}=\left.\frac{d}{d t}\right|_{t=0} \int_{V}\left[j^{1}\left(\sigma_{t} \circ \phi\right)\right]^{*} \Theta_{\mathcal{L}} \\
& =\left.\frac{d}{d t}\right|_{t=0} \int_{V}\left(j^{1} \phi\right)^{*}\left[\left(j^{1} \sigma_{t}\right)^{*} \Theta_{\mathcal{L}}\right]=\int_{V}\left(j^{1} \phi\right)^{*}\left(\lim _{t \rightarrow 0} \frac{\left(j^{1} \sigma_{t}\right)^{*} \Theta_{\mathcal{L}}-\Theta_{\mathcal{L}}}{t}\right) \\
& =\int_{V}\left(j^{1} \phi\right)^{*} \mathrm{~L}\left(j^{1} Z\right) \Theta_{\mathcal{L}}=\int_{V}\left(j^{1} \phi\right)^{*}\left[i\left(j^{1} Z\right) \mathrm{d} \Theta_{\mathcal{L}}+\mathrm{d} i\left(j^{1} Z\right) \Theta_{\mathcal{L}}\right] \\
& =-\int_{V}\left(j^{1} \phi\right)^{*}\left[i\left(j^{1} Z\right) \Omega_{\mathcal{L}}-\mathrm{d} i\left(j^{1} Z\right) \Theta_{\mathcal{L}}\right] \\
& =-\int_{V}\left(j^{1} \phi\right)^{*} i\left(j^{1} Z\right) \Omega_{\mathcal{L}}+\int_{V} \mathrm{~d}\left[\left(j^{1} \phi\right)^{*} i(Z) \Theta_{\mathcal{L}]}\right. \\
& =-\int_{V}\left(j^{1} \phi\right)^{*} i\left(j^{1} Z\right) \Omega_{\mathcal{L}}+\int_{\partial V}\left(j^{1} \phi\right)^{*} i\left(j^{1} Z\right) \Theta_{\mathcal{L}} \\
& =-\int_{V}\left(j^{1} \phi\right)^{*} i\left(j^{1} Z\right) \Omega_{\mathcal{L}},
\end{aligned}
$$

as a consequence of Stoke's theorem and the hypothesis made on the supports of the vertical fields. Thus, by the fundamental theorem of the variational calculus we conclude that $\left.\frac{d}{d t}\right|_{t=0} \int_{V}\left(j^{1} \phi_{t}\right)^{*} \Theta_{\mathcal{L}}=0$ if, and only if, $\left(j^{1} \phi\right)^{*} i\left(j^{1} Z\right) \Omega_{\mathcal{L}}=0$, for every compact-supported $Z \in \mathfrak{X}^{\mathrm{V}(\pi)}(E)$. However, as compact-supported vector fields generate locally the $\mathrm{C}^{\infty}(E)$-module of vector fields in $E$, it follows that the last equality holds for every $Z \in \mathfrak{X}^{\mathrm{V}(\pi)}(E)$.

Now, suppose $\phi \in \Gamma(M, E)$ is a critical section; that is, $\left(j^{1} \phi\right)^{*} i\left(j^{1} Z\right) \Omega_{\mathcal{L}}=0$, for every $Z \in \mathfrak{X}^{\mathrm{V}(\pi)}(E)$, and consider $X \in \mathfrak{X}\left(J^{1} E\right)$, which can be written as $X=X_{\phi}+X_{v}$ where $X_{\phi}$ is tangent to the image of $j^{1} \phi$ and $X_{v}$ is $\bar{\pi}^{1}$-vertical, both in the points of the image of $j^{1} \phi$. However, $X_{v}=\left(X_{v}-j^{1}\left(\pi_{*}^{1} X_{v}\right)\right)+j^{1}\left(\pi_{*}^{1} X_{v}\right)$, where $j^{1}\left(\pi_{*}^{1} X_{v}\right)$ is understood as the prolongation of a vector field which coincides with $\pi_{*}^{1} X_{v}$ on the image of $\phi$. Observe that $\pi_{*}^{1}\left(X_{v}-j^{1}\left(\pi_{*}^{1} X_{v}\right)\right)=0$ on the points of the image of $j^{1} \phi$. Therefore

$$
\left(j^{1} \phi\right)^{*} i(X) \Omega_{\mathcal{L}}=\left(j^{1} \phi\right)^{*} i\left(X_{\phi}\right) \Omega_{\mathcal{L}}+\left(j^{1} \phi\right)^{*} i\left(X_{v}-j^{1}\left(\pi_{*}^{1} X_{v}\right)\right) \Omega_{\mathcal{L}}+\left(j^{1} \phi\right)^{*} i\left(j^{1}\left(\pi_{*}^{1} X_{v}\right)\right) \Omega_{\mathcal{L}}
$$

However, $\left(j^{1} \phi\right)^{*} i\left(X_{\phi}\right) \Omega_{\mathcal{L}}=0$, because $X_{\phi}$ is tangent to the image of $j^{1} \phi$, hence $\Omega_{\mathcal{L}}$ acts on linearly dependent vector fields. Nevertheless, $\left(j^{1} \phi\right)^{*} i\left(X_{v}-j^{1}\left(\pi_{*}^{1} X_{v}\right)\right) \Omega_{\mathcal{L}}=0$, because $X_{v}-$ $j^{1}\left(\pi_{*}^{1} X_{v}\right)$ is $\pi^{1}$-vertical and $\Omega_{\mathcal{L}}$ vanishes on these vector fields, when it is restricted to $j^{1} \phi$. Therefore, as $\phi$ is stationary and $\pi_{*}^{1} X_{v} \in \mathfrak{X}^{\mathrm{V}(\pi)}(E)$, we have

$$
\int_{M}\left(j^{1} \phi\right)^{*} i(X) \Omega_{\mathcal{L}}=\int_{M}\left(j^{1} \phi\right)^{*} i\left(j^{1}\left(\pi_{*}^{1} X_{v}\right)\right) \Omega_{\mathcal{L}}=0 .
$$

The converse is a consequence of the first paragraph, since the condition $\left(j^{1} \phi\right)^{*} i(X) \Omega_{\mathcal{L}}=0$, $\forall X \in \mathfrak{X}\left(J^{1} \pi\right)$, holds, in particular, for $j^{1} Z$, for every $Z \in \mathfrak{X}^{\mathrm{V}(\pi)}(E)$.

$(2 \Leftrightarrow 3)$ If $X=\alpha^{\nu} \frac{\partial}{\partial x^{\nu}}+\beta^{A} \frac{\partial}{\partial y^{A}}+\gamma_{\nu}^{A} \frac{\partial}{\partial v_{\nu}^{A}} \in \mathfrak{X}\left(J^{1} \pi\right)$, taking into account the local expression (1) of $\Omega_{\mathcal{L}}$, we have

$$
\begin{aligned}
i(X) \Omega_{\mathcal{L}}= & (-1)^{\eta} \alpha^{\nu}\left[\frac{\partial^{2} £}{\partial v_{\mu}^{B} \partial v_{\eta}^{A}} \mathrm{~d} v_{\mu}^{B} \wedge \mathrm{d} y^{A} \wedge \mathrm{d}^{m-2} x_{\eta \nu}+\frac{\partial^{2} £}{\partial y^{B} \partial v_{\eta}^{A}} \mathrm{~d} y^{B} \wedge \mathrm{d} y^{A} \wedge \mathrm{d}^{m-2} x_{\eta \nu}\right. \\
& \left.-\frac{\partial^{2} £}{\partial v_{\mu}^{B} \partial v_{\eta}^{A}} v_{\eta}^{A} \mathrm{~d} v_{\mu}^{B} \wedge \mathrm{d}^{m-1} x_{\nu}-\left(\frac{\partial^{2} £}{\partial y^{B} \partial v_{\eta}^{A}} v_{\eta}^{A}-\frac{\partial £}{\partial y^{B}}+\frac{\partial^{2} £}{\partial x^{\eta} \partial v_{\eta}^{B}}\right) \mathrm{d} y^{B} \wedge \mathrm{d}^{m-1} x_{\nu}\right] \\
& +\beta^{A}\left[\frac{\partial^{2} £}{\partial v_{\mu}^{B} \partial v_{\eta}^{A}} \mathrm{~d} v_{\mu}^{B} \wedge \mathrm{d}^{m-1} x_{\eta}+\left(\frac{\partial^{2} £}{\partial y^{A} \partial v_{\eta}^{B}}-\frac{\partial^{2} £}{\partial y^{B} \partial v_{\eta}^{A}}\right) \mathrm{d} y^{B} \wedge \mathrm{d}^{m-1} x_{\eta}\right.
\end{aligned}
$$




$$
\begin{aligned}
& \left.+\left(\frac{\partial^{2} £}{\partial y^{A} \partial v_{\eta}^{B}} v_{\eta}^{B}-\frac{\partial £}{\partial y^{A}}+\frac{\partial^{2} £}{\partial x^{\eta} \partial v_{\eta}^{A}}\right) \mathrm{d}^{m} x\right] \\
& +\gamma_{\nu}^{A}\left[-\frac{\partial^{2} £}{\partial v_{\nu}^{A} \partial v_{\eta}^{B}} \mathrm{~d} y^{B} \wedge \mathrm{d}^{m-1} x_{\eta}+\frac{\partial^{2} £}{\partial v_{\nu}^{A} \partial v_{\eta}^{B}} v_{\eta}^{B} \mathrm{~d}^{m} x\right]
\end{aligned}
$$

but if $\phi=\left(x^{\mu}, y^{A}\left(x^{\eta}\right)\right)$, then $j^{1} \phi=\left(x^{\mu}, y^{A}\left(x^{\eta}\right), v^{A}\left(x^{\eta}\right)\right)=\left(x^{\mu}, y^{A}\left(x^{\eta}\right), \frac{\partial y^{A}}{\partial x^{\mu}}\left(x^{\eta}\right)\right)$, and hence

$$
\begin{aligned}
\left(j^{1} \phi\right)^{*} i(X) \Omega_{\mathcal{L}}= & (-1)^{\eta+\nu} \alpha^{\eta}\left[\frac{\partial}{\partial x^{\mu}}\left(\frac{\partial £}{\partial v_{\mu}^{A}} \circ j^{1} \phi\right)-\frac{\partial £}{\partial y^{A}} \circ j^{1} \phi\right] \frac{\partial\left(y^{A} \circ \phi\right)}{\partial x^{\eta}} \mathrm{d}^{m} x \\
& +\beta^{A}\left[\frac{\partial}{\partial x^{\mu}}\left(\frac{\partial £}{\partial v_{\mu}^{A}} \circ j^{1} \phi\right)-\frac{\partial £}{\partial y^{A}} \circ j^{1} \phi\right] \mathrm{d}^{m} x
\end{aligned}
$$

and, as this holds for every $X \in \mathfrak{X}\left(J^{1} \pi\right)$, we conclude that $\left(j^{1} \phi\right)^{*} i(X) \Omega_{\mathcal{L}}=0$ if, and only if, the Euler-Lagrange equations (4) hold for $\phi$.

$(3 \Leftrightarrow 4) \quad$ Using the local expressions (1) of $\Omega_{h}$ and (3) for $\mathcal{X}_{\mathcal{L}}$, and taking $f=1$ as a representative of the class $\left\{\mathcal{X}_{\mathcal{L}}\right\}$, from the equation (5), we obtain that

$$
\begin{aligned}
& 0=\left(F_{\mu}^{B}-v_{\mu}^{B}\right) \frac{\partial^{2} £}{\partial v_{\nu}^{A} \partial v_{\mu}^{B}}, \\
& 0=\frac{\partial £}{\partial y^{A}}-\frac{\partial^{2} £}{\partial x^{\mu} \partial v_{\mu}^{A}}-\frac{\partial^{2} £}{\partial y^{B} \partial v_{\mu}^{A}} F_{\mu}^{B}-\frac{\partial^{2} £}{\partial v_{\nu}^{B} \partial v_{\mu}^{A}} G_{\nu \mu}^{B}+\frac{\partial^{2} £}{\partial y^{A} \partial v_{\mu}^{B}}\left(F_{\mu}^{B}-v_{\mu}^{B}\right),
\end{aligned}
$$

but, if $X_{\mathcal{L}}$ is holonomic, it is semiholonomic and then $F_{\mu}^{B}=v_{\mu}^{B}$. Therefore the equations (7) are identities, and the equations (8) are

$$
0=\frac{\partial £}{\partial y^{A}}-\frac{\partial^{2} £}{\partial x^{\mu} \partial v_{\mu}^{A}}-\frac{\partial^{2} £}{\partial y^{B} \partial v_{\mu}^{A}} v_{\mu}^{B}-\frac{\partial^{2} £}{\partial v_{\nu}^{B} \partial v_{\mu}^{A}} G_{\nu \mu}^{B} .
$$

Now, for a section $\phi=\left(x^{\mu}, y^{A}\left(x^{\eta}\right)\right)$, if $j^{1} \phi=\left(x^{\mu}, y^{A}\left(x^{\eta}\right), \frac{\partial y^{A}}{\partial x^{\mu}}\left(x^{\eta}\right)\right)$ is an integral section of $X_{\mathcal{L}}$, then $G_{\nu \mu}^{A}=\frac{\partial^{2} y^{A}}{\partial x^{\nu} \partial x^{\mu}}$, and the equations (9) are equivalent to the Euler-Lagrange equations for $\phi$.

$(3 \Leftrightarrow 5)$ The proof is like in the above item: using the local expressions (1) of $\Omega_{\mathcal{L}}$ and $(2)$ for $\nabla_{\mathcal{L}}$, we prove that the equation (6) holds for an integrable connection if, and only if, the Euler-Lagrange equations (4) hold for its integral sections.

Semi-holonomic (but not necessarily integrable) locally decomposable multivector fields and connections which are solution to the Lagrangian equations (5) and (6) respectively are called Euler-Lagrange multivector fields and connections for $\left(J^{1} \pi, \Omega_{\mathcal{L}}\right)$.

If $\left(J^{1} \pi, \Omega_{\mathcal{L}}\right)$ is regular, Euler-Lagrange $m$-multivector fields and connections exist in $J^{1} \pi$, although they are not necessarily integrable. If $\left(J^{1} \pi, \Omega_{\mathcal{L}}\right)$ is singular, in the most favourable cases, Euler-Lagrange multivector fields and connections only exist in some submanifold $S \hookrightarrow J^{1} \pi$, which can be obtained after applying a suitable constraint algorithm (see [70]).

\section{Hamiltonian formalism}

\subsection{Multimomentum bundles. Legendre maps}

As we have pointed out in the introduction, the construction of the Hamiltonian formalism of field theories is more involved than the Lagrangian formulation. In fact, there are different bundles where the Hamiltonian formalism can be developed (see, for instance, [29], and references therein). Here we take one of the most standard choices. 
First, $\mathcal{M} \pi \equiv \Lambda_{2}^{m} \mathrm{~T}^{*} E$, is the bundle of $m$-forms on $E$ vanishing by the action of two $\pi$-vertical vector fields (so $\operatorname{dim} \mathcal{M} \pi=n m+n+m+1$ ), and is diffeomorphic to the set $\operatorname{Aff}\left(J^{1} \pi, \Lambda^{m} \mathrm{~T}^{*} M\right)$, made of the affine maps from $J^{1} \pi$ to $\Lambda^{m} \mathrm{~T}^{*} M$ (the multicotangent bundle of $M$ of order $m$ [15]) $[16,30]$. It is called the extended multimomentum bundle, and its canonical submersions are denoted

$$
\kappa: \mathcal{M} \pi \rightarrow E ; \quad \bar{\kappa}=\pi \circ \kappa: \mathcal{M} \pi \rightarrow M .
$$

As $\mathcal{M} \pi$ is a subbundle of $\Lambda^{m} \mathrm{~T}^{*} E$, then $\mathcal{M} \pi$ is endowed with a canonical form $\Theta \in \Omega^{m}(\mathcal{M} \pi)$ (the "tautological form"), which is defined as follows: let $(x, \alpha) \in \Lambda_{2}^{m} \mathrm{~T}^{*} E$, with $x \in E$ and $\alpha \in \Lambda_{2}^{m} \mathrm{~T}_{x}^{*} E$; then, for every $X_{1}, \ldots, X_{m} \in \mathrm{T}_{(x, \alpha)}(\mathcal{M} \pi)$,

$$
\Theta\left((x, \alpha) ; X_{1}, \ldots, X_{m}\right):=\alpha\left(x ; \mathrm{T}_{(x, \alpha)} \kappa\left(X_{1}\right), \ldots, \mathrm{T}_{(q, \alpha)} \kappa\left(X_{m}\right)\right) .
$$

Then we define the multisymplectic form $\Omega:=-\mathrm{d} \Theta \in \Omega^{m+1}(\mathcal{M} \pi)$. They are known as the multimomentum Liouville $m$ and $(m+1)$-forms

If we introduce natural coordinates $\left(x^{\nu}, y^{A}, p_{A}^{\nu}, p\right)$ in $\mathcal{M} \pi$ adapted to the bundle $\pi: E \rightarrow M$, and such that $\omega=\mathrm{d}^{m} x$, the local expressions of these forms are

$$
\Theta=p_{A}^{\nu} \mathrm{d} y^{A} \wedge \mathrm{d}^{m-1} x_{\nu}+p \mathrm{~d}^{m} x, \quad \Omega=-\mathrm{d} p_{A}^{\nu} \wedge \mathrm{d} y^{A} \wedge \mathrm{d}^{m-1} x_{\nu}-\mathrm{d} p \wedge \mathrm{d}^{m} x .
$$

Now we denote by $J^{1} \pi^{*}$ the quotient $\mathcal{M} \pi / \pi^{*} \Lambda^{m} \mathrm{~T}^{*} M$, with $\operatorname{dim} J^{1} \pi^{*}=n m+n+m$. We have the natural submersions

$$
\tau: J^{1} \pi^{*} \rightarrow E ; \quad \bar{\tau}=\pi \circ \tau: J^{1} \pi^{*} \rightarrow M .
$$

Furthermore, the natural submersion $\mu: \mathcal{M} \pi \rightarrow J^{1} \pi^{*}$ endows $\mathcal{M} \pi$ with the structure of an affine bundle over $J^{1} \pi^{*}$, with $(\pi \circ \tau)^{*} \Lambda^{m} \mathrm{~T}^{*} M$ as the associated vector bundle. $J^{1} \pi^{*}$ is usually called the restricted multimomentum bundle associated with the bundle $\pi: E \rightarrow M$.

Natural coordinates in $J^{1} \pi^{*}$ (adapted to the bundle $\pi: E \rightarrow M$ ) are denoted by $\left(x^{\nu}, y^{A}, p_{A}^{\nu}\right)$.

Definition 3. Let $\left(J^{1} \pi, \Omega_{\mathcal{L}}\right)$ be a Lagrangian system. The extended Legendre map associated with $\mathcal{L}, \widetilde{\mathcal{F} L}: J^{1} \pi \rightarrow \mathcal{M} \pi$, is defined by

$$
(\widetilde{\mathcal{F} L}(\bar{y}))\left(Z_{1}, \ldots, Z_{m}\right):=\left(\Theta_{\mathcal{L}}\right)_{\bar{y}}\left(\bar{Z}_{1}, \ldots, \bar{Z}_{m}\right),
$$

where $Z_{1}, \ldots, Z_{m} \in \mathrm{T}_{\pi^{1}(\bar{y})} E$, and $\bar{Z}_{1}, \ldots, \bar{Z}_{m} \in \mathrm{T}_{\bar{y}} J^{1} \pi$ are such that $\mathrm{T}_{\bar{y}} \pi^{1} \bar{Z}_{\alpha}=Z_{\alpha}$.

The restricted Legendre map associated with $\mathcal{L}$ is $\mathcal{F} \mathcal{L}:=\mu \circ \widetilde{\mathcal{F} L}: J^{1} \pi \rightarrow J^{1} \pi^{*}$.

In natural coordinates we have:

$$
\begin{aligned}
& \widetilde{\mathcal{F} L}^{*} x^{\alpha}=x^{\alpha}, \quad \widetilde{\mathcal{F} L}^{*} y^{A}=y^{A}, \quad \widetilde{\mathcal{F} L}^{*} p_{A}^{\alpha}=\frac{\partial £}{\partial v_{\alpha}^{A}}, \quad \widetilde{\mathcal{F} L}^{*} p=£-v_{\alpha}^{A} \frac{\partial £}{\partial v_{\alpha}^{A}}, \\
& \mathcal{F} \mathcal{L}^{*} x^{\alpha}=x^{\alpha}, \quad \mathcal{F} \mathcal{L}^{*} y^{A}=y^{A}, \quad \mathcal{F} \mathcal{L}^{*} p_{A}^{\alpha}=\frac{\partial £}{\partial v_{\alpha}^{A}} .
\end{aligned}
$$

Then, observe that $\widetilde{\mathcal{F} \mathcal{L}}{ }^{*} \Theta=\Theta_{\mathcal{L}}$, and $\widetilde{\mathcal{F} \mathcal{L}}{ }^{*} \Omega=\Omega_{\mathcal{L}}$.

Definition 4. $\left(J^{1} \pi, \Omega_{\mathcal{L}}\right)$ is regular (hyper-regular) if $\mathcal{F} \mathcal{L}$ is a local (global) diffeomorphism. Elsewhere it is singular. (This definition is equivalent to that given above.)

$\left(J^{1} \pi, \Omega_{\mathcal{L}}\right)$ is almost-regular if

1. $\mathcal{P}:=\mathcal{F} \mathcal{L}\left(J^{1} \pi\right)$ is a closed submanifold of $J^{1} \pi^{*}$ (natural embedding $\jmath_{0}: \mathcal{P} \hookrightarrow J^{1} \pi^{*}$ ).

2. $\mathcal{F} \mathcal{L}$ is a submersion onto its image.

3. The fibres $\mathcal{F} \mathcal{L}^{-1}(\mathcal{F} \mathcal{L}(\bar{y})), \forall \bar{y} \in J^{1} \pi$, are connected submanifolds of $J^{1} \pi$. 


\subsection{The (hyper)regular case}

In the Hamiltonian formalism of field theories, there are different ways of introducing the physical information (the "Hamiltonian"). For instance, we can use connections in the multimomentum bundles in order to obtain a covariant definition of the so-called Hamiltonian densities (see, for instance, [16, 39, 79, 91]).

Nevertheless, the simplest way of defining (regular) Hamiltonian systems in field theory consists in considering the bundle $\bar{\tau}: J^{1} \pi^{*} \rightarrow M$ and then giving sections $h: J^{1} \pi^{*} \rightarrow \mathcal{M} \pi$ of the projection $\mu$, which are called Hamiltonian sections and carry the physical information of the system. Then we can define the differentiable forms

$$
\Theta_{h}:=h^{*} \Theta \in \Omega^{m}\left(J^{1} \pi^{*}\right), \quad \Omega_{h}:=-\mathrm{d} \Theta_{h}=h^{*} \Omega \in \Omega^{m+1}\left(J^{1} \pi^{*}\right)
$$

which are the Hamilton-Cartan $m$ and $(m+1)$ forms of $J^{1} \pi^{*}$ associated with the Hamiltonian section $h$. The couple $\left(J^{1} \pi^{*}, \Omega_{h}\right)$ is said to be a Hamiltonian system.

In a local chart of natural coordinates, a Hamiltonian section is specified by a local Hamiltonian function $\mathrm{h} \in \mathrm{C}^{\infty}(U), U \subset J^{1} \pi^{*}$, such that $h\left(x^{\nu}, y^{A}, p_{A}^{\nu}\right) \equiv\left(x^{\nu}, y^{A}, p_{A}^{\nu}, p=-\mathrm{h}\left(x^{\gamma}, y^{B}, p_{B}^{\eta}\right)\right)$. Then, the local expressions of the Hamilton-Cartan forms associated with $h$ are

$$
\Theta_{h}=p_{A}^{\nu} \mathrm{d} y^{A} \wedge \mathrm{d}^{m-1} x_{\nu}-\mathrm{hd}^{m} x, \quad \Omega_{h}=-\mathrm{d} p_{A}^{\nu} \wedge \mathrm{d} y^{A} \wedge \mathrm{d}^{m-1} x_{\nu}+\mathrm{dh} \wedge \mathrm{d}^{m} x .
$$

Notice that $\Omega_{h}$ is 1-nondegenerate; that is, a multisymplectic form (as a simple calculation in coordinates shows).

Now we want to associate Hamiltonian systems to the Lagrangian ones. First we consider the hyper-regular case (the regular case is analogous, but working locally).

If $\left(J^{1} \pi, \Omega_{\mathcal{L}}\right)$ is a hyper-regular Lagrangian system, then we have the diagram

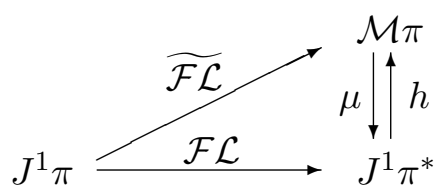

It is proved [16] that $\tilde{\mathcal{P}}:=\widetilde{\mathcal{F} \mathcal{L}}\left(J^{1} \pi\right)$ is a 1-codimensional imbedded submanifold of $\mathcal{M} \pi\left(\tilde{J}_{0}: \tilde{\mathcal{P}} \hookrightarrow\right.$ $\mathcal{M} \pi$ denotes is the natural embedding), which is transverse to $\mu$, and is diffeomorphic to $J^{1} \pi^{*}$. This diffeomorphism is $\mu^{-1}$, when $\mu$ is restricted to $\tilde{\mathcal{P}}$, and also coincides with the map $h:=$ $\widetilde{\mathcal{F} \mathcal{L}} \circ \mathcal{F} \mathcal{L}^{-1}$, when it is restricted onto its image (which is just $\left.\tilde{\mathcal{P}}\right)$. Thus $h$ and $\left(J^{1} \pi^{*}, \Omega_{h}\right)$ are the Hamiltonian section and the Hamiltonian system associated with the hyper-regular Lagrangian system $\left(J^{1} \pi, \Omega_{\mathcal{L}}\right)$, respectively.

Locally, the Hamiltonian section $h\left(x^{\nu}, y^{A}, p_{A}^{\nu}\right)=\left(x^{\nu}, y^{A}, p_{A}^{\nu}, p=-\mathrm{h}\left(x^{\gamma}, y^{B}, p_{B}^{\gamma}\right)\right)$ is specified by the local Hamiltonian function

$$
\mathrm{h}=p_{A}^{\nu}\left(\mathcal{F} \mathcal{L}^{-1}\right)^{*} v_{\nu}^{A}-\left(\mathcal{F} \mathcal{L}^{-1}\right)^{*} £ .
$$

Then we have the local expressions (10) for the corresponding Hamilton-Cartan forms and, of course, $\mathcal{F} \mathcal{L}^{*} \Theta_{h}=\Theta_{\mathcal{L}}$, and $\mathcal{F} \mathcal{L}^{*} \Omega_{h}=\Omega_{\mathcal{L}}$.

The Hamiltonian field equations can also be derived from a variational principle. In fact:

Definition 5. Let $\left(J^{1} \pi^{*}, \Omega_{h}\right)$ be a Hamiltonian system. Let $\Gamma\left(M, J^{1} \pi^{*}\right)$ be the set of sections of $\bar{\tau}$. Consider the map

$$
\begin{array}{cl}
\mathbf{H}: & \Gamma\left(M, J^{1} \pi^{*}\right) \longrightarrow \mathbb{R}, \\
\psi \mapsto \int_{M} \psi^{*} \Theta_{h},
\end{array}
$$


where the convergence of the integral is assumed. The variational problem for this Hamiltonian system is the search for the critical (or stationary) sections of the functional $\mathbf{H}$, with respect to the variations of $\psi$ given by $\psi_{t}=\sigma_{t} \circ \psi$, where $\left\{\sigma_{t}\right\}$ is the local one-parameter group of any compact-supported $Z \in \mathfrak{X}^{\mathrm{V}(\bar{\tau})}\left(J^{1} \pi^{*}\right)$ ( the module of $\bar{\tau}$-vertical vector fields in $J^{1} \pi^{*}$ ), that is:

$$
\left.\frac{\mathrm{d}}{\mathrm{d} t}\right|_{t=0} \int_{M} \psi_{t}^{*} \Theta_{h}=0
$$

This is the so-called Hamilton-Jacobi principle of the Hamiltonian formalism.

The Hamilton-Jacobi principle is equivalent to find distributions $\mathcal{D}$ of $J^{1} \pi^{*}$ such that:

1. $\mathcal{D}$ is $m$-dimensional.

2. $\mathcal{D}$ is $\bar{\tau}$-transverse.

3. $\mathcal{D}$ is integrable (that is, involutive).

4. The integral manifolds of $\mathcal{D}$ are the critical sections of the Hamilton-Jacobi principle.

As in the Lagrangian formalism, $\mathcal{D}$ are associated with classes of integrable and $\bar{\tau}$-transverse $m$-multivector fields $\{\mathcal{X}\} \subset \mathfrak{X}^{m}\left(J^{1} \pi^{*}\right)$ or, what is equivalent, with connections in the bundle $\bar{\pi}: J^{1} \pi \rightarrow M$, whose expressions are

$$
\begin{aligned}
& \mathcal{X}=\bigwedge_{\nu=1}^{m} f\left(\frac{\partial}{\partial x^{\nu}}+F_{\nu}^{A} \frac{\partial}{\partial y^{A}}+G_{A \nu}^{\rho} \frac{\partial}{\partial p_{A}^{\rho}}\right), \quad\left(f \in \mathrm{C}^{\infty}\left(J^{1} \pi^{*}\right) \text { non-vanishing }\right), \\
& \nabla=\mathrm{d} x^{\mu} \otimes\left(\frac{\partial}{\partial x^{\mu}}+F_{\mu}^{A} \frac{\partial}{\partial y^{A}}+G_{A \mu}^{\rho} \frac{\partial}{\partial p_{A}^{\rho}}\right) .
\end{aligned}
$$

Then we have:

Theorem 2. The following assertions on a section $\psi \in \Gamma\left(M, J^{1} \pi^{*}\right)$ are equivalent:

1. $\psi$ is a critical section for the variational problem posed by the Hamilton-Jacobi principle.

2. $\psi^{*} i(X) \Omega_{h}=0, \forall X \in \mathfrak{X}\left(J^{1} \pi^{*}\right)$.

3. If $\left(U ; x^{\nu}, y^{A}, p_{A}^{\nu}\right)$ is a natural system of coordinates in $J^{1} \pi^{*}$, then $\psi$ satisfies the HamiltonDe Donder-Weyl equations in $U$

$$
\frac{\partial\left(y^{A} \circ \psi\right)}{\partial x^{\nu}}=\frac{\partial \mathrm{h}}{\partial p_{A}^{\nu}} \circ \psi, \quad \frac{\partial\left(p_{A}^{\nu} \circ \psi\right)}{\partial x^{\nu}}=-\frac{\partial \mathrm{h}}{\partial y^{A}} \circ \psi .
$$

4. $\psi$ is an integral section of a class of integrable and $\bar{\tau}$-transverse multivector fields $\left\{\mathcal{X}_{h}\right\} \subset$ $\mathfrak{X}^{m}\left(J^{1} \pi^{*}\right)$ satisfying that

$$
i\left(\mathcal{X}_{h}\right) \Omega_{h}=0, \quad \forall \mathcal{X}_{h} \in\left\{\mathcal{X}_{h}\right\}
$$

5. $\psi$ is an integral section of an integrable connection $\nabla_{h}$ in $J^{1} \pi^{*}$ satisfying the equation

$$
i\left(\nabla_{h}\right) \Omega_{h}=(m-1) \Omega_{h} .
$$

Proof. This proof is taken from [23, 28], and [30].

$(1 \Leftrightarrow 2) \quad$ Let $Z \in \mathfrak{X}^{\mathrm{V}(\bar{\tau})}\left(J^{1} \pi^{*}\right)$ be a compact-supported vector field, and $V \subset M$ an open set such that $\partial V$ is a $(m-1)$-dimensional manifold and that $\bar{\tau}(\operatorname{supp}(Z)) \subset V$. Then

$$
\left.\frac{d}{d t}\right|_{t=0} \int_{M} \psi_{t}^{*} \Theta_{h}=\left.\frac{d}{d t}\right|_{t=0} \int_{V} \psi_{t}^{*} \Theta_{h}=\left.\frac{d}{d t}\right|_{t=0} \int_{V} \psi^{*}\left(\sigma_{t}^{*} \Theta_{h}\right)=\int_{V} \psi^{*}\left(\lim _{t \rightarrow 0} \frac{\sigma_{t}^{*} \Theta_{h}-\Theta_{h}}{t}\right)
$$




$$
\begin{aligned}
& =\int_{V} \psi^{*} \mathrm{~L}(Z) \Theta_{h}=\int_{V} \psi^{*}\left(i(Z) \mathrm{d} \Theta_{h}+\mathrm{d} i(Z) \Theta_{h}\right) \\
& =-\int_{V} \psi^{*}\left(i(Z) \Omega_{h}-\mathrm{d} i(Z) \Theta_{h}\right)=-\int_{V} \psi^{*} i(Z) \Omega_{h}+\int_{V} \mathrm{~d}\left[\psi^{*} i(Z) \Theta_{h}\right] \\
& =-\int_{V} \psi^{*} i(Z) \Omega_{h}+\int_{\partial V} \psi^{*} i(Z) \Theta_{h}=-\int_{V} \psi^{*} i(Z) \Omega_{h},
\end{aligned}
$$

as a consequence of Stoke's theorem and the hypothesis made on the supports of the vertical fields. Thus, by the fundamental theorem of the variational calculus we conclude that $\left.\frac{d}{d t}\right|_{t=0} \int_{V} \psi_{t}^{*} \Theta_{h}=0$ if, and only if, $\psi^{*} i(Z) \Omega_{h}=0$, for every compact-supported $Z \in \mathfrak{X}^{\mathrm{V}(\bar{\tau})}\left(J^{1} \pi^{*}\right)$. However, as compact-supported vector fields generate locally the $\mathrm{C}^{\infty}\left(J^{1} \pi^{*}\right)$-module of vector fields in $J^{1} \pi^{*}$, it follows that the last equality holds for every $Z \in \mathfrak{X}^{\mathrm{V}(\bar{\tau})}\left(J^{1} \pi^{*}\right)$.

Now, if $\mathrm{p} \in \operatorname{Im} \psi$, then $\mathrm{T}_{\mathrm{p}} J^{1} \pi^{*}=\mathrm{V}_{\mathrm{p}}(\bar{\tau}) \oplus \mathrm{T}_{\mathrm{p}}(\operatorname{Im} \psi)$. So if $X \in \mathfrak{X}\left(J^{1} \pi^{*}\right)$, then

$$
X_{\mathrm{p}}=\left(X_{\mathrm{p}}-\mathrm{T}_{\mathrm{p}}(\psi \circ \bar{\tau})\left(X_{\mathrm{p}}\right)\right)+\mathrm{T}_{\mathrm{p}}(\psi \circ \bar{\tau})\left(X_{\mathrm{p}}\right) \equiv X_{\mathrm{p}}^{V}+X_{\mathrm{p}}^{\psi},
$$

and therefore

$$
\psi^{*} i(X) \Omega_{h}=\psi^{*} i\left(X^{V}\right) \Omega_{h}+\psi^{*} i\left(X^{\psi}\right) \Omega_{h}=\psi^{*} i\left(X^{\psi}\right) \Omega_{h}=0,
$$

since $\psi^{*} i\left(X^{V}\right) \Omega_{h}=0$, by the conclusion in the above paragraph. Furthermore, $X_{\mathrm{p}}^{\psi} \in \mathrm{T}_{\mathrm{p}}(\operatorname{Im} \psi)$, and $\operatorname{dim}(\operatorname{Im} \psi)=m$, being $\Omega_{h} \in \Omega^{m+1}\left(J^{1} \pi^{*}\right)$. Hence we conclude that $\psi^{*} i(X) \Omega_{h}=0$, for every $X \in \mathfrak{X}\left(J^{1} \pi^{*}\right)$.

The converse is obvious taking into account the reasoning of the first paragraph, since the condition $\psi^{*} i(X) \Omega_{h}=0, \forall X \in \mathfrak{X}\left(J^{1} \pi^{*}\right)$, holds, in particular, for every $Z \in \mathfrak{X}^{\mathrm{V}(\bar{\tau})}\left(J^{1} \pi^{*}\right)$.

$(2 \Leftrightarrow 3)$ If $X=\alpha^{\nu} \frac{\partial}{\partial x^{\nu}}+\beta^{A} \frac{\partial}{\partial y^{A}}+\gamma_{A}^{\nu} \frac{\partial}{\partial p_{A}^{\nu}} \in \mathfrak{X}\left(J^{1} \pi^{*}\right)$, taking into account the local expression $(10)$ of $\Omega_{h}$, we have

$$
\begin{aligned}
i(X) \Omega_{h}= & (-1)^{\eta} \alpha^{\eta}\left(\mathrm{d} p_{A}^{\nu} \wedge \mathrm{d} y^{A} \wedge \mathrm{d}^{m-2} x_{\eta \nu}-\frac{\partial \mathrm{h}}{\partial p_{A}^{\nu}} \mathrm{d} p_{A}^{\nu} \wedge \mathrm{d}^{m-1} x_{\eta}\right) \\
& +\beta^{A}\left(\mathrm{~d} p_{A}^{\nu} \wedge \mathrm{d}^{m-1} x_{\nu}+\frac{\partial \mathrm{h}}{\partial y^{A}} \mathrm{~d}^{m} x\right)+\gamma_{A}^{\nu}\left(-\mathrm{d} y^{A} \wedge \mathrm{d}^{m-1} x_{\nu}+\frac{\partial \mathrm{h}}{\partial p_{A}^{\nu}} \wedge \mathrm{d}^{m} x\right)
\end{aligned}
$$

but if $\psi=\left(x^{\nu}, y^{A}\left(x^{\eta}\right), p_{A}^{\nu}\left(x^{\eta}\right)\right)$, then

$$
\begin{aligned}
\psi^{*} i(X) \Omega_{h}= & (-1)^{\eta+\nu} \alpha^{\eta}\left(\frac{\partial\left(y^{A} \circ \psi\right)}{\partial x^{\nu}}-\left.\frac{\partial \mathrm{h}}{\partial p_{A}^{\nu}}\right|_{\psi}\right) \frac{\partial\left(p_{A}^{\nu} \circ \psi\right)}{\partial x^{\eta}} \mathrm{d}^{m} x \\
& +\beta^{A}\left(\frac{\partial\left(p_{A}^{\nu} \circ \psi\right)}{\partial x^{\nu}}+\left.\frac{\partial \mathrm{h}}{\partial y^{A}}\right|_{\psi}\right) \mathrm{d}^{m} x+\gamma_{A}^{\nu}\left(-\frac{\partial\left(y^{A} \circ \psi\right)}{\partial x^{\nu}}+\left.\frac{\partial \mathrm{h}}{\partial p_{A}^{\nu}}\right|_{\psi}\right) \mathrm{d}^{m} x
\end{aligned}
$$

and, as this holds for every $X \in \mathfrak{X}\left(J^{1} \pi^{*}\right)$, we conclude that $\psi^{*} i(X) \Omega_{h}=0$ if, and only if, the Hamilton-De Donder-Weyl equations (13) hold for $\psi$.

$(3 \Leftrightarrow 4)$ Using the local expressions (10) of $\Omega_{h}$ and (11) for $\mathcal{X}_{h}$, and taking $f=1$ as a representative of the class $\left\{\mathcal{X}_{h}\right\}$, the equation (14), in coordinates, is

$$
F_{\nu}^{A}=\frac{\partial \mathrm{h}}{\partial p_{A}^{\nu}}, \quad G_{A \nu}^{\nu}=-\frac{\partial \mathrm{h}}{\partial y^{A}} .
$$

This result allows us to assure the local existence of (classes of) multivector fields satisfying the desired conditions. The corresponding global solutions are then obtained using a partition of unity subordinated to a covering of $J^{1} \pi^{*}$ made of local natural charts. Now, if $\psi(x)=$ $\left(x^{\nu}, y^{A}\left(x^{\gamma}\right), p_{A}^{\nu}\left(x^{\gamma}\right)\right)$ is an integral section of $\mathcal{X}_{h}$, then

$$
\frac{\partial\left(y^{A} \circ \psi\right)}{\partial x^{\nu}}=F_{\nu}^{A} \circ \psi, \quad \frac{\partial\left(p_{A}^{\rho} \circ \psi\right)}{\partial x^{\nu}}=G_{A \nu}^{\rho} \circ \psi .
$$


Thus, combining both expressions we obtain the Hamilton-De Donder-Weyl equations (13) for $\psi$.

$(3 \Leftrightarrow 5) \quad$ The proof is like in the above item: using the local expressions (10) of $\Omega_{h}$ and (12) for $\nabla_{h}$, we prove that the equation (15) holds for an integrable connection if, and only if, the Hamilton-De Donder-Weyl equations (13) hold for its integral sections.

The $\bar{\tau}$-transverse locally decomposable multivector fields and connections which are solution to the Hamiltonian equations (14) and (15) respectively (but not necessarily integrable) are called Hamilton-De Donder-Weyl multivector fields and connections for $\left(J^{1} \pi^{*}, \Omega_{h}\right)$.

Hence, the existence of Hamilton-De Donder-Weyl multivector fields and connections for $\left(J^{1} \pi^{*}, \Omega_{h}\right)$ is assured, although they are not necessarily integrable.

Finally, we can establish the equivalence between the Lagrangian and Hamiltonian formalisms in the hyper-regular case:

Theorem 3. (equivalence theorem for sections) Let $\left(J^{1} \pi, \Omega_{\mathcal{L}}\right)$ be a hyper-regular Lagrangian system, and $\left(J^{1} \pi^{*}, \Omega_{h}\right)$ the associated Hamiltonian system.

If a section $\phi \in \Gamma(M, E)$ is a solution to the Lagrangian variational problem (Hamilton principle), then the section $\psi=\mathcal{F} \mathcal{L} \circ j^{1} \phi \in \Gamma\left(M, J^{1} \pi^{*}\right)$ is a solution to the Hamiltonian variational problem (Hamilton-Jacobi principle).

Conversely, if $\psi \in \Gamma\left(M, J^{1} \pi^{*}\right)$ is a solution to the Hamiltonian variational problem, then the section $\phi=\tau \circ \psi \in \Gamma(M, E)$ is a solution to the Lagrangian variational problem.

Proof. This proof is taken from [28] and [30].

Bearing in mind the diagram

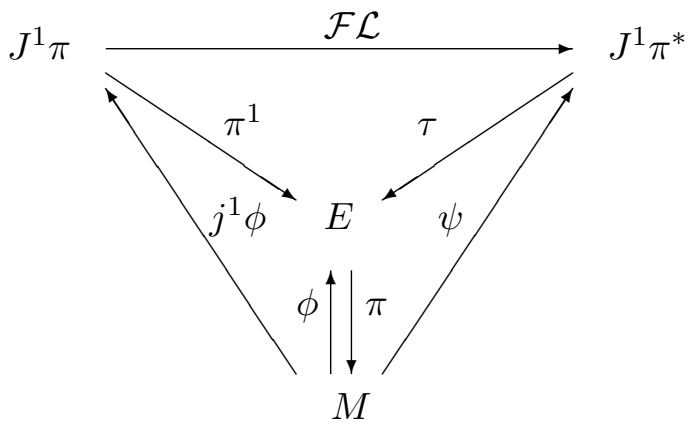

if $\phi$ is a solution to the Lagrangian variational problem then $\left(j^{1} \phi\right)^{*} i(X) \Omega_{\mathcal{L}}=0$, for every $X \in \mathfrak{X}\left(J^{1} \pi\right)$ (Theorem 1, item 2); therefore, as $\mathcal{F} \mathcal{L}$ is a local diffeomorphism,

$$
\begin{aligned}
0 & =\left(j^{1} \phi\right)^{*} i(X) \Omega_{\mathcal{L}}=\left(j^{1} \phi\right)^{*} i(X)\left(\mathcal{F} \mathcal{L}^{*} \Omega_{h}\right) \\
& \left.=\left(j^{1} \phi\right)^{*} \mathcal{F} \mathcal{L}^{*}\left(i\left(\mathcal{F} \mathcal{L}_{*}^{-1} X\right) \Omega_{h}\right)=\left(\mathcal{F} \mathcal{L} \circ j^{1} \phi\right)^{*} i\left(X^{\prime}\right) \Omega_{h}\right),
\end{aligned}
$$

which holds for every $X^{\prime} \in \mathfrak{X}\left(J^{1} \pi^{*}\right)$ and thus, by the item 2 of Theorem $2, \psi \equiv \mathcal{F} \mathcal{L} \circ j^{1} \phi$ is a solution to the Hamiltonian variational problem.

Conversely, let $\psi \in \Gamma\left(M, J^{1} \pi^{*}\right)$ be a solution to the Hamiltonian variational problem. Reversing the above reasoning we obtain that $\left(\mathcal{F} \mathcal{L}^{-1} \circ \psi\right)^{*} i(X) \Omega_{\mathcal{L}}=0$, for every $X \in \mathfrak{X}\left(J^{1} \pi\right)$, and hence $\sigma \equiv \mathcal{F} \mathcal{L}^{-1} \circ \psi \in \Gamma\left(M, J^{1} E\right)$ is a critical section for the Lagrangian variational problem. Then, as we are in the hyper-regular case, $\sigma$ must be an holonomic section, $\sigma=j^{1} \phi[27,69,92]$, and since the above diagram is commutative, $\phi=\tau^{1} \circ \psi \in \Gamma(M, E)$.

The equivalence between the Lagrangian and the Hamiltonian formalisms can be stated also in terms of multivector fields and connections (see [28]). 


\subsection{The almost-regular case}

Now, consider the almost-regular case. Let $\tilde{\mathcal{P}}:=\widetilde{\mathcal{F}} \mathcal{L}\left(J^{1} \pi\right), \mathcal{P}:=\mathcal{F} \mathcal{L}\left(J^{1} \pi\right)$ (the natural projections are denoted by $\tau_{0}^{1}: \mathcal{P} \rightarrow E$ and $\left.\bar{\tau}_{0}^{1}:=\pi \circ \tau_{0}^{1}: \mathcal{P} \rightarrow M\right)$, and assume that $\mathcal{P}$ is a fibre bundle over $E$ and $M$. Denote by $\tilde{\jmath}_{0}: \tilde{\mathcal{P}} \hookrightarrow \mathcal{M} \pi$ the natural imbedding, and by $\widetilde{\mathcal{F}}_{0}$ and $\mathcal{F} \mathcal{L}_{0}$ the restrictions of $\widetilde{\mathcal{F} \mathcal{L}}$ and $\mathcal{F} \mathcal{L}$ to their images, respectively. So, we have the diagram

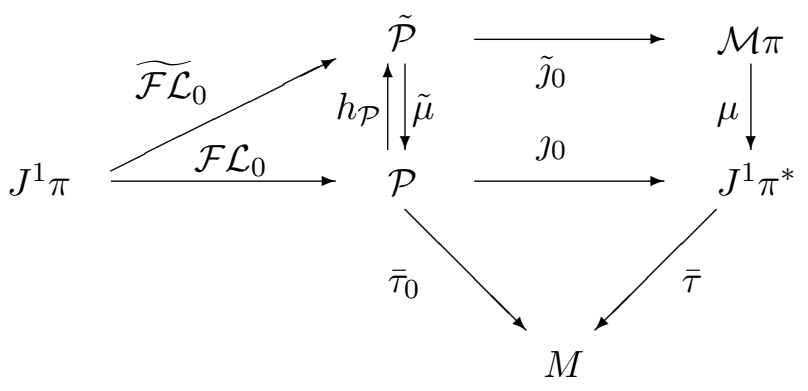

Now, it can be proved that the $\mu$-transverse submanifold $\tilde{\mathcal{P}}$ is diffeomorphic to $\mathcal{P}$ [69]. This diffeomorphism is denoted $\tilde{\mu}: \tilde{\mathcal{P}} \rightarrow \mathcal{P}$, and it is just the restriction of the projection $\mu$ to $\tilde{\mathcal{P}}$. Then, taking $h_{\mathcal{P}}:=\tilde{\mu}^{-1}$, we define the Hamilton-Cartan forms

$$
\Theta_{h}^{0}=\left(\tilde{\jmath}_{0} \circ h_{\mathcal{P}}\right)^{*} \Theta \in \Omega^{m}(\mathcal{P}), \quad \Omega_{h}^{0}=-\mathrm{d} \Theta_{h}^{0}\left(\tilde{\jmath}_{0} \circ h_{\mathcal{P}}\right)^{*} \Omega \in \Omega^{m+1}(\mathcal{P}),
$$

which verify that $\mathcal{F} \mathcal{L}_{0}^{*} \Omega_{h}^{0}=\Omega_{\mathcal{L}}$. Then $h_{\mathcal{P}}$ is also called a Hamiltonian section, and $\left(\mathcal{P}, \Omega_{h}^{0}\right)$ is the Hamiltonian system associated with the almost-regular Lagrangian system $\left(J^{1} \pi, \Omega_{\mathcal{L}}\right)$. In general, $\Omega_{h}^{0}$ is a pre-multisymplectic form and $\left(\mathcal{P}, \Omega_{h}^{0}\right)$ is the Hamiltonian system associated with the almost-regular Lagrangian system $\left(J^{1} \pi, \Omega_{\mathcal{L}}\right)$.

In this framework, the Hamilton-Jacobi principle for $\left(\mathcal{P}, \Omega_{h}^{0}\right)$ is stated like above, and the critical sections $\psi_{0} \in \Gamma(M, \mathcal{P})$ can be characterized in an analogous way than in Theorem 2 .

If $\Omega_{h}^{0}$ is a pre-multisymplectic form, Hamilton-De Donder-Weyl multivector vector fields and connections only exist, in the most favourable cases, in some submanifold $S \hookrightarrow J^{1} \pi$, and they are not necessarily integrable. As in the Lagrangian case, $S$ can be obtained after applying the suitable constraint algorithm [70]. Then, the equivalence theorem follows in an analogous way than above.

It is important to point out that the analysis of the Hamiltonian description of non-regular field theories is far to be completed and, in fact, there is a lot of topics under discussion. For instance, there are some kinds of singular Lagrangian systems for which the construction of the associated Hamiltonian formalism (following the procedure that we have presented here) is ambiguous and, in order to overcome this trouble, a different notion of regularity must be done, which involve the use of Lepagean forms [64, 66, 67]. Neverthelees, the analysis of this and other problems exceeds the scope of this work.

\section{Unified Lagrangian-Hamiltonian formalism}

\subsection{Geometric framework}

The extended and the restricted jet-multimomentum bundles are

$$
\mathcal{W}:=J^{1} \pi \times_{E} \mathcal{M} \pi, \quad \mathcal{W}_{r}:=J^{1} \pi \times_{E} J^{1} \pi^{*},
$$

with natural coordinates $\left(x^{\alpha}, y^{A}, v_{\alpha}^{A}, p_{A}^{\alpha}, p\right)$ and $\left(x^{\alpha}, y^{A}, v_{\alpha}^{A}, p_{A}^{\alpha}\right)$. We have natural projections (submersions) $\mu_{\mathcal{W}}: \mathcal{W} \rightarrow \mathcal{W}_{r}$, and

$$
\begin{array}{llll}
\rho_{1}: \mathcal{W} \rightarrow J^{1} \pi, & \rho_{2}: \mathcal{W} \rightarrow \mathcal{M} \pi, & \rho_{E}: \mathcal{W} \rightarrow E, & \rho_{M}: \mathcal{W} \rightarrow M, \\
\rho_{1}^{r}: \mathcal{W}_{r} \rightarrow J^{1} \pi, & \rho_{2}^{r}: \mathcal{W}_{r} \rightarrow J^{1} \pi^{*}, & \rho_{E}^{r}: \mathcal{W}_{r} \rightarrow E, & \rho_{M}^{r}: \mathcal{W}_{r} \rightarrow M .
\end{array}
$$


Definition 6. The coupling $m$-form in $\mathcal{W}$, denoted by $\mathcal{C}$, is an $m$-form along $\rho_{M}$ which is defined as follows: for every $\bar{y} \in J_{y}^{1} E$, with $\bar{\pi}^{1}(\bar{y})=\pi(y)=x \in E$, and $\mathbf{p} \in \mathcal{M}_{y} \pi$, let $w \equiv(\bar{y}, \mathbf{p}) \in \mathcal{W}_{y}$, then

$$
\mathcal{C}(w):=\left(\mathrm{T}_{x} \phi\right)^{*} \mathbf{p}
$$

where $\phi: M \rightarrow E$ satisfies that $j^{1} \phi(x)=\bar{y}$. Then, we denote by $\hat{\mathcal{C}} \in \Omega^{m}(\mathcal{W})$ the $\rho_{M}$-semibasic form associated with $\mathcal{C}$.

The canonical $m$-form $\Theta_{\mathcal{W}} \in \Omega^{m}(\mathcal{W})$ is defined as $\Theta_{\mathcal{W}}:=\rho_{2}^{*} \Theta$, and is $\rho_{E}$-semibasic. The canonical $(m+1)$-form is the pre-multisymplectic form $\Omega_{\mathcal{W}}:=-\mathrm{d} \Theta_{\mathcal{W}}=\rho_{1}^{*} \Omega \in \Omega^{m+1}(\mathcal{W})$.

There exists $\hat{C} \in \mathrm{C}^{\infty}(\mathcal{W})$ such that $\hat{\mathcal{C}}=\hat{C}\left(\rho_{M}^{*} \omega\right)$, and $\hat{\mathcal{C}}(w)=\left(p+p_{A}^{\alpha} v_{\alpha}^{A}\right) \mathrm{d}^{m} x$.

Local expressions of $\Theta_{\mathcal{W}}$ and $\Omega_{\mathcal{W}}$ are the same than for $\Theta$ and $\Omega$.

Let $\hat{\mathcal{L}}:=\rho_{1}^{*} \mathcal{L} \in \Omega^{m}(\mathcal{W})$, and $\hat{\mathcal{L}}=\hat{L}\left(\rho_{M}^{*} \omega\right)$, with $\hat{L}=\rho_{1}^{*} L \in \mathrm{C}^{\infty}(\mathcal{W})$. We define the Hamiltonian submanifold $\jmath_{0}: \mathcal{W}_{0} \hookrightarrow \mathcal{W}$ by

$$
\mathcal{W}_{0}:=\{w \in \mathcal{W} \mid \hat{\mathcal{L}}(w)=\hat{\mathcal{C}}(w)\}
$$

The constraint function defining $\mathcal{W}_{0}$ is

$$
\hat{C}-\hat{L}=p+p_{A}^{\alpha} v_{\alpha}^{A}-\hat{L}\left(x^{\nu}, y^{B}, v_{\nu}^{B}\right)=0 .
$$

There are projections which are the restrictions to $\mathcal{W}_{0}$ of the projections (16), as it is shown in the following diagram:

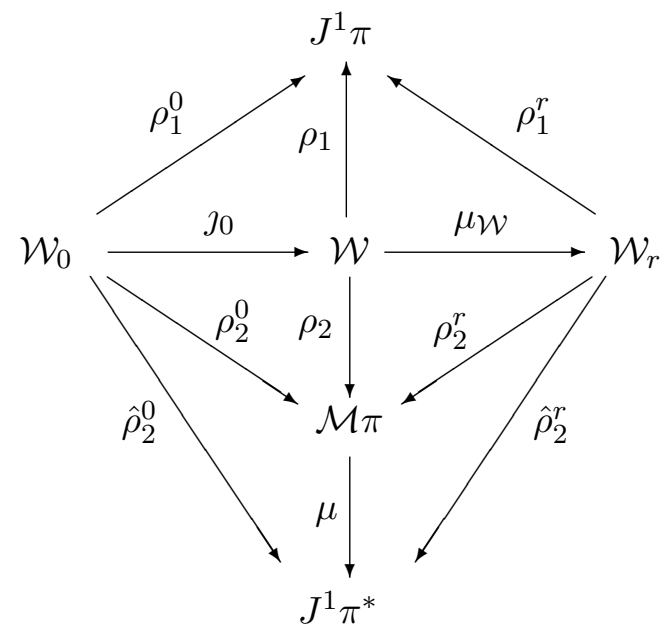

$\left(x^{\alpha}, y^{A}, v_{\alpha}^{A}, p_{A}^{\alpha}\right)$ are local coordinates in $\mathcal{W}_{0}$, and

$$
\begin{array}{ll}
\rho_{1}^{0}\left(x^{\alpha}, y^{A}, v_{\alpha}^{A}, p_{A}^{\alpha}\right)=\left(x^{\alpha}, y^{A}, v_{\alpha}^{A}\right), & \jmath_{0}\left(x^{\alpha}, y^{A}, v_{\alpha}^{A}, p_{A}^{\alpha}\right)=\left(x^{\alpha}, y^{A}, v_{\alpha}^{A}, p_{A}^{\alpha}, L-v_{\alpha}^{A} p_{A}^{\alpha}\right), \\
\hat{\rho}_{2}^{0}\left(x^{\alpha}, y^{A}, v_{\alpha}^{A}, p_{A}^{\alpha}\right)=\left(x^{\alpha}, y^{A}, p_{A}^{\alpha}\right), & \rho_{2}^{0}\left(x^{\alpha}, y^{A}, v_{\alpha}^{A}, p_{A}^{\alpha}\right)=\left(x^{\alpha}, y^{A}, p_{A}^{\alpha}, L-v_{\alpha}^{A} p_{A}^{\alpha}\right) .
\end{array}
$$

It is proved that $\mathcal{W}_{0}$ is a 1 -codimensional $\mu_{\mathcal{W}}$-transversal submanifold of $\mathcal{W}$, diffeomorphic to $\mathcal{W}_{r}$. As a consequence, $\mathcal{W}_{0}$ induces a Hamiltonian section of $\mu_{\mathcal{W}}, \hat{h}: \mathcal{W}_{r} \rightarrow \mathcal{W}$, which is locally specified by giving the local Hamiltonian function $\hat{H}=-\hat{L}+p_{A}^{\alpha} v_{\alpha}^{A}$; that is, $\hat{h}\left(x^{\alpha}, y^{A}, v_{\alpha}^{A}, p_{A}^{\alpha}\right)=$ $\left(x^{\alpha}, y^{A}, v_{\alpha}^{A}, p_{A}^{\alpha},-\hat{H}\right)$. From $\hat{h}$ we recover a Hamiltonian section $\tilde{h}: \mathcal{P} \rightarrow \mathcal{M} \pi$ defined by $\tilde{h}([\mathbf{p}])=$ $\left(\rho_{2} \circ \hat{h}\right)\left[\left(\rho_{2}^{r}\right)^{-1}(\jmath([\mathbf{p}]))\right], \forall[\mathbf{p}] \in \mathcal{P}$. (See the diagram.)

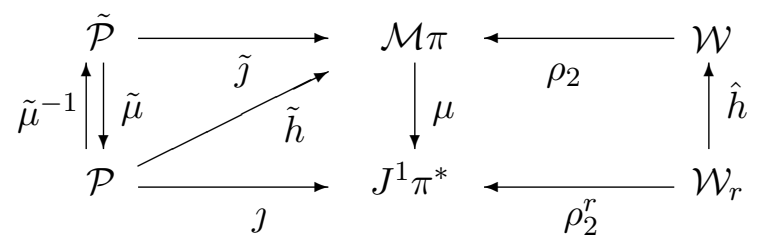


(For hyper-regular systems we have $\tilde{\mathcal{P}}=\mathcal{M} \pi$ and $\mathcal{P}=J^{1} \pi^{*}$.)

We define the forms $\Theta_{0}:=\jmath_{0}^{*} \Theta_{\mathcal{W}}=\rho_{2}^{0 *} \Theta \in \Omega^{m}\left(\mathcal{W}_{0}\right)$, and $\Omega_{0}:=\jmath_{0}^{*} \Omega_{\mathcal{W}}=\rho_{2}^{0 *} \Omega \in \Omega^{m+1}\left(\mathcal{W}_{0}\right)$, whose local expressions are

$$
\begin{aligned}
& \Theta_{0}=\left(L-p_{A}^{\alpha} v_{\alpha}^{A}\right) \mathrm{d}^{m} x+p_{A}^{\alpha} \mathrm{d} y^{A} \wedge \mathrm{d}^{m-1} x_{\alpha}, \\
& \Omega_{0}=\mathrm{d}\left(p_{A}^{\alpha} v_{\alpha}^{A}-L\right) \wedge \mathrm{d}^{m} x-\mathrm{d} p_{A}^{\alpha} \wedge \mathrm{d} y^{A} \wedge \mathrm{d}^{m-1} x_{\alpha},
\end{aligned}
$$

$\left(\mathcal{W}_{0}, \Omega_{0}\right)$ (equiv. $\left.\left(\mathcal{W}_{r}, \hat{h}^{*} \Omega_{0}\right)\right)$ is a pre-multisymplectic Hamiltonian system.

\subsection{Field equations}

A Lagrange-Hamilton problem consists in finding sections $\psi_{0} \in \Gamma\left(M, \mathcal{W}_{0}\right)$ such that

$$
\psi_{0}^{*} i\left(Y_{0}\right) \Omega_{0}=0, \quad \forall Y_{0} \in \mathfrak{X}\left(\mathcal{W}_{0}\right) .
$$

Taking $Y_{0} \in \mathfrak{X}^{\mathrm{V}\left(\hat{\rho}_{2}^{0}\right)}\left(\mathcal{W}_{0}\right)$ we get the first constraint submanifold $\jmath_{1}: \mathcal{W}_{1} \hookrightarrow \mathcal{W}_{0}$,

$$
\mathcal{W}_{1}=\left\{(\bar{y}, \mathbf{p}) \in \mathcal{W}_{0} \mid i\left(V_{0}\right)\left(\Omega_{0}\right)_{(\bar{y}, \mathbf{p})}=0, \text { for every } V_{0} \in \mathrm{V}\left(\hat{\rho}_{2}^{0}\right)\right\},
$$

and sections solution to (17) take values on it. $\mathcal{W}_{1}$ is defined by $p_{A}^{\alpha}=\frac{\partial L}{\partial v_{\alpha}^{A}}$, hence

$$
\mathcal{W}_{1}=\left\{(\bar{y}, \widetilde{\mathcal{F}} \mathcal{L}(\bar{y})) \in \mathcal{W} \mid \bar{y} \in J^{1} \pi\right\},
$$

and $\mathcal{W}_{1}$ is diffeomorphic to $J^{1} \pi$.

Theorem 4. (see diagram (18)) If $\psi_{0}: M \rightarrow \mathcal{W}_{0}$ is a section fulfilling equation (17), then $\psi_{0}=\left(\psi_{\mathcal{L}}, \psi_{\mathcal{H}}\right)=\left(\psi_{\mathcal{L}}, \widetilde{\mathcal{F}} \mathcal{L} \circ \psi_{\mathcal{L}}\right)$, where $\psi_{\mathcal{L}}=\rho_{1}^{0} \circ \psi_{0}$, and:

1. $\psi_{\mathcal{L}}$ is the canonical lift of the projected section $\phi=\rho_{E}^{0} \circ \psi_{0}: M \rightarrow E$ (that is, $\psi_{\mathcal{L}}$ is a holonomic section).

2. $\psi_{\mathcal{L}}=j^{1} \phi$ is a solution to the Lagrangian problem, and $\mu \circ \psi_{\mathcal{H}}=\mu \circ \widetilde{\mathcal{F} L} \circ \psi_{\mathcal{L}}=\mathcal{F} \mathcal{L} \circ j^{1} \phi$ is a solution to the Hamiltonian problem.

Conversely, for every section $\phi: M \rightarrow E$ such that $j^{1} \phi$ is a solution to the Lagrangian problem (and hence $\mathcal{F} \mathcal{L} \circ j^{1} \phi$ is a solution to the Hamiltonian problem) we have that $\psi_{0}=\left(j^{1} \phi, \widetilde{\mathcal{F} \mathcal{L}} \circ j^{1} \phi\right)$, is a solution to (17).

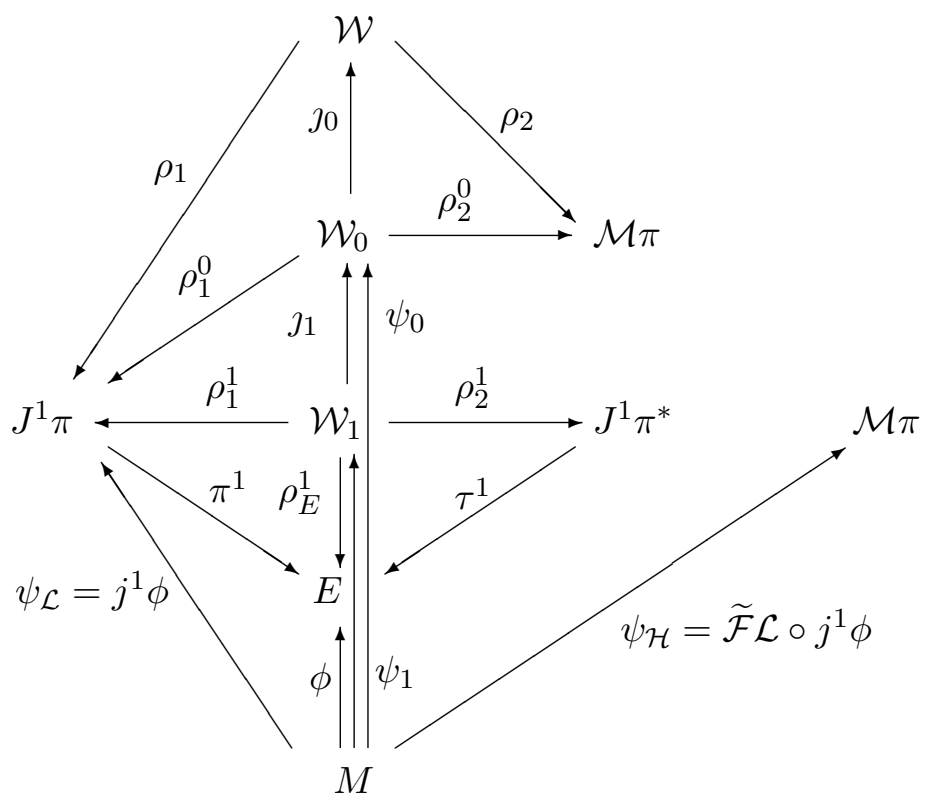


Proof. This proof is taken from [24]. See also [71].

1. Taking $\left\{\frac{\partial}{\partial p_{A}^{\alpha}}\right\}$ as a local basis for the $\rho_{1}^{0}$-vertical vector fields, and a section $\psi_{0}$, we have

$$
\begin{aligned}
& i\left(\frac{\partial}{\partial p_{A}^{\alpha}}\right) \Omega_{0}=v_{\alpha}^{A} \mathrm{~d}^{m} x-\mathrm{d} y^{A} \wedge \mathrm{d}^{m-1} x_{\alpha} \Longrightarrow \\
& 0=\psi_{0}^{*}\left[i\left(\frac{\partial}{\partial p_{A}^{\alpha}}\right) \Omega_{0}\right]=\left(v_{\alpha}^{A}(x)-\frac{\partial y^{A}}{\partial x^{\alpha}}\right) \mathrm{d}^{m} x,
\end{aligned}
$$

and thus the holonomy condition appears naturally within the unified formalism, and it is not necessary to impose it by hand to $\psi_{0}$. Thus we have that $\psi_{0}=\left(x^{\alpha}, y^{A}, \frac{\partial y^{A}}{\partial x^{\alpha}}, \frac{\partial L}{\partial v_{\alpha}^{A}}\right)$, since $\psi_{0}$ takes values in $\mathcal{W}_{1}$, and hence it is of the form $\psi_{0}=\left(j^{1} \phi, \widetilde{\mathcal{F} \mathcal{L}} \circ j^{1} \phi\right)$, for $\phi=\left(x^{\alpha}, y^{A}\right)=\rho_{E}^{0} \circ \psi_{0}$.

2. Since sections $\psi_{0}: M \rightarrow \mathcal{W}_{0}$ solution to (17) take values in $\mathcal{W}_{1}$, we can identify them with sections $\psi_{1}: M \rightarrow \mathcal{W}_{1}$. These sections $\psi_{1}$ verify, in particular, that $\psi_{1}^{*} i\left(Y_{1}\right) \Omega_{1}=0$ holds for every $Y_{1} \in \mathfrak{X}\left(\mathcal{W}_{1}\right)$. Obviously $\psi_{0}=\jmath_{1} \circ \psi_{1}$. Moreover, as $\mathcal{W}_{1}$ is the graph of $\widetilde{\mathcal{F} L}$, denoting by $\rho_{1}^{1}=\rho_{1}^{0} \circ \jmath_{1}: \mathcal{W}_{1} \rightarrow J^{1} \pi$ the diffeomorphism which identifies $\mathcal{W}_{1}$ with $J^{1} \pi$, if we define $\Omega_{1}=\jmath_{1}^{*} \Omega_{0}$, we have that $\Omega_{1}=\rho_{1}^{1 *} \Omega_{\mathcal{L}}$. In fact; as $\left(\rho_{1}^{1}\right)^{-1}(\bar{y})=(\bar{y}, \widetilde{\mathcal{F} L}(\bar{y}))$, for every $\bar{y} \in J^{1} \pi$, then $\left(\rho_{0}^{2} \circ \jmath_{1} \circ\left(\rho_{1}^{1}\right)^{-1}\right)(\bar{y})=\widetilde{\mathcal{F} L}(\bar{y}) \in \mathcal{M} \pi$, and hence

$$
\Omega_{\mathcal{L}}=\left(\rho_{0}^{2} \circ \jmath_{1} \circ\left(\rho_{1}^{1}\right)^{-1}\right)^{*} \Omega=\left[\left(\left(\rho_{1}^{1}\right)^{-1}\right)^{*} \circ \jmath_{1}^{*} \circ \rho_{0}^{2 *}\right] \Omega=\left[\left(\left(\rho_{1}^{1}\right)^{-1}\right)^{*} \circ \jmath_{1}^{*}\right] \Omega_{0}=\left(\left(\rho_{1}^{1}\right)^{-1}\right)^{*} \Omega_{1} .
$$

Now, let $X \in \mathfrak{X}\left(J^{1} \pi\right)$. We have

$$
\begin{aligned}
\left(j^{1} \phi\right)^{*} i(X) \Omega_{\mathcal{L}} & =\left(\rho_{1}^{0} \circ \psi_{0}\right)^{*} i(X) \Omega_{\mathcal{L}}=\left(\rho_{1}^{0} \circ \jmath_{1} \circ \psi_{1}\right)^{*} i(X) \Omega_{\mathcal{L}} \\
& =\left(\rho_{1}^{1} \circ \psi_{1}\right)^{*} i(X) \Omega_{\mathcal{L}}=\psi_{1}^{*} i\left(\left(\rho_{1}^{1}\right)_{*}^{-1} X\right)\left(\rho_{1}^{1 *} \Omega_{\mathcal{L}}\right)=\psi_{1}^{*} i\left(Y_{1}\right) \Omega_{1} \\
& =\psi_{1}^{*} i\left(Y_{1}\right)\left(\jmath_{1}^{*} \Omega_{0}\right)=\left(\psi_{1}^{*} \circ \jmath_{1}^{*}\right) i\left(Y_{0}\right) \Omega_{0}=\psi_{0}^{*} i\left(Y_{0}\right) \Omega_{0},
\end{aligned}
$$

where $Y_{0} \in \mathfrak{X}\left(\mathcal{W}_{0}\right)$ is such that $Y_{0}=\jmath_{1 *} Y_{1}$. But as $\psi_{0}^{*} i\left(Y_{0}\right) \Omega_{0}=0$, for every $Y_{0} \in \mathfrak{X}\left(\mathcal{W}_{0}\right)$, then we conclude that $\left(j^{1} \phi\right)^{*} i(X) \Omega_{\mathcal{L}}=0$, for every $X \in \mathfrak{X}\left(J^{1} \pi\right)$.

Conversely, let $j^{1} \phi: M \rightarrow J^{1} \pi$ such that $\left(j^{1} \phi\right)^{*} i(X) \Omega_{\mathcal{L}}=0$, for every $X \in \mathfrak{X}\left(J^{1} \pi\right)$, and define $\psi_{0}: M \rightarrow \mathcal{W}_{0}$ as $\psi_{0}:=\left(j^{1} \phi, \widetilde{\mathcal{F} \mathcal{L}} \circ j^{1} \phi\right)$ (observe that $\psi_{0}$ takes its values in $\mathcal{W}_{1}$ ). Taking into account that, on the points of $\mathcal{W}_{1}$, every $Y_{0} \in \mathfrak{X}\left(\mathcal{W}_{0}\right)$ splits into $Y_{0}=Y_{0}^{1}+Y_{0}^{2}$, with $Y_{0}^{1} \in \mathfrak{X}\left(\mathcal{W}_{0}\right)$ tangent to $\mathcal{W}_{1}$, and $Y_{0}^{2} \in \mathfrak{X}^{\mathrm{V}\left(\rho_{1}^{0}\right)}\left(\mathcal{W}_{0}\right)$, we have that

$$
\psi_{0}^{*} i\left(Y_{0}\right) \Omega_{0}=\psi_{0}^{*} i\left(Y_{0}^{1}\right) \Omega_{0}+\psi_{0}^{*} i\left(Y_{0}^{2}\right) \Omega_{0}=0,
$$

because for $Y_{0}^{1}$, the same reasoning as in (19) leads to

$$
\psi_{0}^{*} i\left(Y_{0}^{1}\right) \Omega_{0}=\left(j^{1} \phi\right)^{*} i\left(X_{0}^{1}\right) \Omega_{\mathcal{L}}=0,
$$

(where $X_{0}^{1}=\left(\rho_{1}^{1}\right)_{*}^{-1} Y_{0}^{1}$ ) and, as $j^{1} \phi$ is a holonomic section for $Y_{0}^{2}$, following also the same reasoning as in (19), a local calculus gives

$$
\psi_{0}^{*} i\left(Y_{0}^{2}\right) \Omega_{0}=\left(j^{1} \phi\right)^{*}\left[\left(f_{A}^{\alpha}(x)\left(v_{\alpha}^{A}-\frac{\partial y^{A}}{\partial x^{\alpha}}\right)\right) \mathrm{d}^{m} x\right]=0 .
$$

The result for the sections $\mathcal{F} \mathcal{L} \circ j^{1} \phi$ is a direct consequence of the equivalence Theorem 3 between the Lagrangian and Hamiltonian formalisms.

Thus, equation (17) gives equations of three different classes:

1. Algebraic equations, determining $\mathcal{W}_{1} \hookrightarrow \mathcal{W}_{0}$, where the sections solution take their values. These are the primary Hamiltonian constraints, and generate, by $\hat{\rho}_{2}^{0}$ projection, the primary constraints of the Hamiltonian formalism for singular Lagrangians. 
2. Differential equations, forcing the sections solution $\psi_{0}$ to be holonomic.

3. The Euler-Lagrange equations.

Field equations in the unified formalism can also be stated in terms of multivector fields and connections in $\mathcal{W}_{0}$. In fact, the problem of finding sections solution to (17) can be formulated equivalently as follows: finding a distribution $D_{0}$ of $\mathrm{T}\left(\mathcal{W}_{0}\right)$ such that it is integrable (that is, involutive), $m$-dimensional, $\rho_{M}^{0}$-transverse, and the integral manifolds of $D_{0}$ are the sections solution to the above equations. (Note that we do not ask them to be lifting of $\pi$-sections; that is, the holonomic condition). This is equivalent to stating that the sections solution to this problem are the integral sections of one of the following equivalent elements:

- A class of integrable and $\rho_{M}^{0}$-transverse $m$-multivector fields $\left\{X_{0}\right\} \subset \mathfrak{X}^{m}\left(\mathcal{W}_{0}\right)$ satisfying that

$$
i\left(X_{0}\right) \Omega_{0}=0, \quad \text { for every } X_{0} \in\left\{X_{0}\right\} .
$$

- An integrable connection $\nabla_{0}$ in $\rho_{M}^{0}: \mathcal{W}_{0} \rightarrow M$ such that

$$
i\left(\nabla_{0}\right) \Omega_{0}=(m-1) \Omega_{0}
$$

Locally decomposable and $\rho_{M}^{0}$-transverse multivector fields and orientable connections which are solutions of these equations are called Lagrange-Hamiltonian multivector fields and jet fields for $\left(\mathcal{W}_{0}, \Omega_{0}\right)$. Euler-Lagrange and Hamilton-De Donder-Weyl multivector fields can be recovered from these Lagrange-Hamiltonian multivector fields (see [24]).

\section{Example}

As an example of application of these formalisms we consider a classical system which has been taken from [24]: minimal surfaces (in $\mathbb{R}^{3}$ ). Other examples of application of the multisymplectic formalism are explained in detail in $[39,43,91]$ as well as in many other references (see, for instance, $[16,25,26,27,28,30,69,71]$ and quoted references).

\subsection{Geometric elements. Lagrangian and Hamiltonian formalisms}

The problem consists in looking for mappings $\varphi: U \subset \mathbb{R}^{2} \rightarrow \mathbb{R}$ such that their graphs have minimal area as sets of $\mathbb{R}^{3}$, and satisfy certain boundary conditions.

For this model, we have that $M=\mathbb{R}^{2}, E=\mathbb{R}^{2} \times \mathbb{R}$, and

$$
\begin{aligned}
& J^{1} \pi=\pi^{*} \mathrm{~T}^{*} \mathbb{R}^{2} \otimes \mathbb{R}=\pi^{*} \mathrm{~T}^{*} M=\pi^{*} \mathrm{~T}^{*} \mathbb{R}^{2}, \quad \mathcal{M} \pi=\pi^{*}\left(\mathrm{~T} M \times_{M} E\right), \\
& J^{1} \pi^{*}=\pi^{*} \mathrm{~T} M=\pi^{*} \mathbb{T}^{2} .
\end{aligned}
$$

The coordinates in $J^{1} \pi, J^{1} \pi^{*}$ and $\mathcal{M} \pi$ are denoted $\left(x^{1}, x^{2}, y, v_{1}, v_{2}\right),\left(x^{1}, x^{2}, y, p^{1}, p^{2}\right)$, and $\left(x^{1}, x^{2}, y, p^{1}, p^{2}, p\right)$ respectively. If $\omega=\mathrm{d} x^{1} \wedge \mathrm{d} x^{2}$, the Lagrangian density is

$$
\mathcal{L}=\left[1+\left(v_{1}\right)^{2}+\left(v_{2}\right)^{2}\right]^{1 / 2} \mathrm{~d} x^{1} \wedge \mathrm{d} x^{2} \equiv £ \mathrm{~d} x^{1} \wedge \mathrm{d} x^{2},
$$

and the Poincaré-Cartan forms are

$$
\begin{aligned}
& \Theta_{\mathcal{L}}=\frac{v_{1}}{£} \mathrm{~d} y \wedge \mathrm{d} x^{2}-\frac{v_{2}}{£} \mathrm{~d} y \wedge \mathrm{d} x^{1}+£\left(1-\left(\frac{v_{1}}{£}\right)^{2}-\left(\frac{v_{2}}{£}\right)^{2}\right) \mathrm{d} x^{1} \wedge \mathrm{d} x^{2}, \\
& \Omega_{\mathcal{L}}=-\mathrm{d}\left(\frac{v_{1}}{£}\right) \wedge \mathrm{d} y \wedge \mathrm{d} x^{2}+\mathrm{d}\left(\frac{v_{2}}{£}\right) \wedge \mathrm{d} y \wedge \mathrm{d} x^{1}
\end{aligned}
$$




$$
-\mathrm{d}\left[£\left(1-\left(\frac{v_{1}}{£}\right)^{2}-\left(\frac{v_{2}}{£}\right)^{2}\right)\right] \wedge \mathrm{d} x^{1} \wedge \mathrm{d} x^{2}
$$

The Euler-Lagrange equation of the problem are

$$
\begin{aligned}
0= & \left(\frac{\partial p^{2}}{\partial x^{2}}+\frac{\partial p^{1}}{\partial x^{1}}\right) \mathrm{d} x^{1} \wedge \mathrm{d} x^{2}=\left[\frac{\partial}{\partial x^{1}}\left(\frac{v_{1}}{£}\right)+\frac{\partial}{\partial x^{2}}\left(\frac{v_{2}}{£}\right)\right] \mathrm{d} x^{1} \wedge \mathrm{d} x^{2} \\
= & \frac{1}{£^{3}}\left[\left(1+\left(\frac{\partial y}{\partial x^{1}}\right)^{2}\right) \frac{\partial^{2} y}{\partial x^{2} \partial x^{2}}+\left(1+\left(\frac{\partial y}{\partial x^{2}}\right)^{2}\right) \frac{\partial^{2} y}{\partial x^{1} \partial x^{1}}\right. \\
& \left.-2 \frac{\partial y}{\partial x^{1}} \frac{\partial y}{\partial x^{2}} \frac{\partial^{2} y}{\partial x^{1} \partial x^{2}}\right] \mathrm{d} x^{1} \wedge \mathrm{d} x^{2},
\end{aligned}
$$

and the associated Euler-Lagrange $m$-vector fields and connections which are the solutions to the Lagrangian problem are

$$
\begin{aligned}
X_{\mathcal{L}}= & f\left(\frac{\partial}{\partial x^{1}}+v_{1} \frac{\partial}{\partial y}+\frac{\partial v_{1}}{\partial x^{1}} \frac{\partial}{\partial v_{1}}+\frac{\partial v_{2}}{\partial x^{1}} \frac{\partial}{\partial v_{2}}\right) \wedge\left(\frac{\partial}{\partial x^{2}}+v_{2} \frac{\partial}{\partial y}+\frac{\partial v_{1}}{\partial x^{2}} \frac{\partial}{\partial v_{1}}+\frac{\partial v_{2}}{\partial x^{2}} \frac{\partial}{\partial v_{2}}\right), \\
\nabla_{\mathcal{L}}= & \mathrm{d} x^{1} \otimes\left(\frac{\partial}{\partial x^{1}}+v_{1} \frac{\partial}{\partial y}+\frac{\partial v_{1}}{\partial x^{1}} \frac{\partial}{\partial v_{1}}+\frac{\partial v_{2}}{\partial x^{1}} \frac{\partial}{\partial v_{2}}\right) \\
& +\mathrm{d} x^{2} \otimes\left(\frac{\partial}{\partial x^{2}}+v_{2} \frac{\partial}{\partial y}+\frac{\partial v_{1}}{\partial x^{2}} \frac{\partial}{\partial v_{1}}+\frac{\partial v_{2}}{\partial x^{2}} \frac{\partial}{\partial v_{2}}\right) .
\end{aligned}
$$

The Legendre maps are given by

$$
\begin{aligned}
& \mathcal{F} \mathcal{L}\left(x^{1}, x^{2}, y, v_{1}, v_{2}\right)=\left(x^{1}, x^{2}, y, \frac{v_{1}}{£}, \frac{v_{2}}{£}\right), \\
& \widetilde{\mathcal{F} L}\left(x^{1}, x^{2}, y, v_{1}, v_{2}\right)=\left(x^{1}, x^{2}, y, \frac{v_{1}}{£}, \frac{v_{2}}{£}, £-\frac{\left(v_{1}\right)^{2}}{£}-\frac{\left(v_{2}\right)^{2}}{£}\right),
\end{aligned}
$$

and then $\mathcal{L}$ is hyperregular. The Hamiltonian function is $\mathrm{h}=-\left[1-\left(p^{1}\right)^{2}-\left(p^{2}\right)^{2}\right]^{1 / 2}$, and so the Hamilton-Cartan forms are

$$
\begin{aligned}
& \Theta_{h}=p^{1} \mathrm{~d} y \wedge \mathrm{d} x^{2}-p^{2} \mathrm{~d} y \wedge \mathrm{d} x^{1}-\mathrm{hd} x^{1} \wedge \mathrm{d} x^{2}, \\
& \Omega_{h}=-\mathrm{d} p^{1} \wedge \mathrm{d} y \wedge \mathrm{d} x^{2}+\mathrm{d} p^{2} \wedge \mathrm{d} y \wedge \mathrm{d} x^{1}+\mathrm{dh} \wedge \mathrm{d} x^{1} \wedge \mathrm{d} x^{2} .
\end{aligned}
$$

The Hamilton-De Donder-Weyl equations of the problem are

$$
\frac{\partial y}{\partial x^{1}}=-\frac{p^{1}}{\mathrm{~h}}, \quad \frac{\partial y}{\partial x^{2}}=-\frac{p^{2}}{\mathrm{~h}}, \quad \frac{\partial p^{1}}{\partial x^{1}}=-\frac{\partial p^{2}}{\partial x^{2}},
$$

and the corresponding Hamilton-De Donder-Weyl $m$-vector fields and connections which are the solutions to the Hamiltonian problem are

$$
\begin{aligned}
X_{h}= & f\left(\frac{\partial}{\partial x^{1}}-\frac{p^{1}}{\mathrm{~h}} \frac{\partial}{\partial y}+\frac{\partial p^{1}}{\partial x^{1}} \frac{\partial}{\partial p^{1}}+\frac{\partial p^{2}}{\partial x^{1}} \frac{\partial}{\partial p^{2}}\right) \wedge\left(\frac{\partial}{\partial x^{2}}-\frac{p^{2}}{\mathrm{~h}} \frac{\partial}{\partial y}+\frac{\partial p^{1}}{\partial x^{2}} \frac{\partial}{\partial p^{1}}+\frac{\partial p^{2}}{\partial x^{2}} \frac{\partial}{\partial p^{2}}\right), \\
\nabla_{h}= & \mathrm{d} x^{1} \otimes\left(\frac{\partial}{\partial x^{1}}-\frac{p^{1}}{\mathrm{~h}} \frac{\partial}{\partial y}+\frac{\partial p^{1}}{\partial x^{1}} \frac{\partial}{\partial p^{1}}+\frac{\partial p^{2}}{\partial x^{1}} \frac{\partial}{\partial p^{2}}\right) \\
& +\mathrm{d} x^{2} \otimes\left(\frac{\partial}{\partial x^{2}}-\frac{p^{2}}{\mathrm{~h}} \frac{\partial}{\partial y}+\frac{\partial p^{1}}{\partial x^{2}} \frac{\partial}{\partial p^{1}}+\frac{\partial p^{2}}{\partial x^{2}} \frac{\partial}{\partial p^{2}}\right) .
\end{aligned}
$$




\subsection{Unified formalism}

For the unified formalism we have

$$
\mathcal{W}=\pi^{*} \mathrm{~T}^{*} M \times_{E} \pi^{*}\left(\mathrm{~T} M \times_{M} E\right), \quad \mathcal{W}_{r}=\pi^{*} \mathrm{~T}^{*} M \times_{E} \pi^{*} \mathrm{~T} M=\pi^{*}\left(\mathrm{~T}^{*} M \times_{M} \mathrm{~T} M\right) .
$$

If $w=\left(x^{1}, x^{2}, y, v_{1}, v_{2}, p^{1}, p^{2}, p\right) \in \mathcal{W}$, the coupling form is $\hat{\mathcal{C}}=\left(p^{1} v_{1}+p^{2} v_{2}+p\right) \mathrm{d} x^{1} \wedge \mathrm{d} x^{2}$; therefore

$$
\mathcal{W}_{0}=\left\{\left(x^{1}, x^{2}, y, v_{1}, v_{2}, p^{1}, p^{2}, p\right) \in \mathcal{W} \mid\left[1+\left(v_{1}\right)^{2}+\left(v_{2}\right)^{2}\right]^{1 / 2}-p^{1} v_{1}-p^{2} v_{2}-p=0\right\},
$$

and we have the forms

$$
\begin{aligned}
\Theta_{0}= & \left(\left[1+\left(v_{1}\right)^{2}+\left(v_{2}\right)^{2}\right]^{1 / 2}-p^{1} v_{1}-p^{2} v_{2}\right) \mathrm{d} x^{1} \wedge \mathrm{d} x^{2}-p^{2} \mathrm{~d} y \wedge \mathrm{d} x_{1}+p^{1} \mathrm{~d} y \wedge \mathrm{d} x_{2}, \\
\Omega_{0}= & -\mathrm{d}\left(\left[1+\left(v_{1}\right)^{2}+\left(v_{2}\right)^{2}\right]^{1 / 2}-p^{1} v_{1}-p^{2} v_{2}\right) \wedge \mathrm{d} x^{1} \wedge \mathrm{d} x^{2} \\
& +\mathrm{d} p^{2} \wedge \mathrm{d} y \wedge \mathrm{d} x_{1}-\mathrm{d} p^{1} \wedge \mathrm{d} y \wedge \mathrm{d} x_{2} .
\end{aligned}
$$

Taking first $\hat{\rho}_{2}^{0}$-vertical vector fields $\frac{\partial}{\partial v_{\alpha}}$ we obtain

$$
0=i\left(\frac{\partial}{\partial v_{\alpha}}\right) \Omega_{0}=\left(p^{\alpha}-\frac{v_{\alpha}}{£}\right) \mathrm{d} x^{1} \wedge \mathrm{d} x^{2},
$$

which determines the submanifold $\mathcal{W}_{1}=\operatorname{graph} \widetilde{\mathcal{F} L}$ (diffeomorphic to $J^{1} \pi$ ), and reproduces the expression of the Legendre map. Now, taking $\rho_{1}^{0}$-vertical vector fields $\frac{\partial}{\partial p^{\alpha}}$, the contraction $i\left(\frac{\partial}{\partial p^{\alpha}}\right) \Omega_{0}$ gives, for $\alpha=1,2, v_{1} \mathrm{~d} x^{1} \wedge \mathrm{d} x^{2}-\mathrm{d} y \wedge \mathrm{d} x^{2}$ and $v_{2} \mathrm{~d} x^{1} \wedge \mathrm{d} x^{2}+\mathrm{d} y \wedge \mathrm{d} x^{1}$ respectively, so that, for a section $\psi_{0}=\left(x^{1}, x^{2}, y\left(x^{1}, x^{2}\right), v_{1}\left(x^{1}, x^{2}\right), v_{2}\left(x^{1}, x^{2}\right), p^{1}\left(x^{1}, x^{2}\right), p^{2}\left(x^{1}, x^{2}\right)\right)$ taking values in $\mathcal{W}_{1}$, we have that the condition $\psi_{0}^{*}\left[i\left(\frac{\partial}{\partial p^{\alpha}}\right) \Omega_{0}\right]=0$ leads to

$$
\left(v_{1}-\frac{\partial y}{\partial x^{1}}\right) \mathrm{d} x^{1} \wedge \mathrm{d} x^{2}=0, \quad\left(v_{2}-\frac{\partial y}{\partial x^{2}}\right) \mathrm{d} x^{1} \wedge \mathrm{d} x^{2}=0
$$

which are the holonomy condition. Finally, taking the vector field $\frac{\partial}{\partial y}$ we have

$$
i\left(\frac{\partial}{\partial y}\right) \Omega_{0}=-\mathrm{d} p^{2} \wedge \mathrm{d} x^{1}+\mathrm{d} p^{1} \wedge \mathrm{d} x^{2}
$$

and, for a section $\psi_{0}$ fulfilling the former conditions, the equation $0=\psi_{0}^{*}\left[i\left(\frac{\partial}{\partial y}\right) \Omega_{0}\right]$ leads to the Euler-Lagrange equations (20). Now, bearing in mind the expressions of $h$ and the Legendre map, from the Euler-Lagrange equations we get the Hamilton-De Donder-Weyl equations (21).

The $m$-vector fields and connections which are the solutions to the problem in the unified formalism are

$$
\begin{aligned}
& X_{0}= f\left(\frac{\partial}{\partial x^{1}}+v_{1} \frac{\partial}{\partial y}+\frac{\partial v_{1}}{\partial x^{1}} \frac{\partial}{\partial v_{1}}+\frac{\partial v_{2}}{\partial x^{1}} \frac{\partial}{\partial v_{2}}+\frac{\partial p^{1}}{\partial x^{1}} \frac{\partial}{\partial p^{1}}+\frac{\partial p^{2}}{\partial x^{1}} \frac{\partial}{\partial p^{2}}\right) \\
& \wedge\left(\frac{\partial}{\partial x^{2}}+v_{2} \frac{\partial}{\partial y}+\frac{\partial v_{1}}{\partial x^{2}} \frac{\partial}{\partial v_{1}}+\frac{\partial v_{2}}{\partial x^{2}} \frac{\partial}{\partial v_{2}}+\frac{\partial p^{1}}{\partial x^{2}} \frac{\partial}{\partial p^{1}}+\frac{\partial p^{2}}{\partial x^{2}} \frac{\partial}{\partial p^{2}}\right), \\
& \nabla_{0}= \mathrm{d} x^{1} \otimes\left(\frac{\partial}{\partial x^{1}}+v_{1} \frac{\partial}{\partial y}+\frac{\partial v_{1}}{\partial x^{1}} \frac{\partial}{\partial v_{1}}+\frac{\partial v_{2}}{\partial x^{1}} \frac{\partial}{\partial v_{2}}+\frac{\partial p^{1}}{\partial x^{1}} \frac{\partial}{\partial p^{1}}+\frac{\partial p^{2}}{\partial x^{1}} \frac{\partial}{\partial p^{2}}\right) \\
&+\mathrm{d} x^{2} \otimes\left(\frac{\partial}{\partial x^{2}}+v_{2} \frac{\partial}{\partial y}+\frac{\partial v_{1}}{\partial x^{2}} \frac{\partial}{\partial v_{1}}+\frac{\partial v_{2}}{\partial x^{2}} \frac{\partial}{\partial v_{2}}+\frac{\partial p^{1}}{\partial x^{2}} \frac{\partial}{\partial p^{1}}+\frac{\partial p^{2}}{\partial x^{2}} \frac{\partial}{\partial p^{2}}\right),
\end{aligned}
$$


( $f$ being a non-vanishing function) where the coefficients $\frac{\partial v_{\alpha}}{\partial x^{\nu}}=\frac{\partial^{2} y}{\partial x^{\nu} \partial x^{\alpha}}$ are related by the Euler-Lagrange equations, and the coefficients $\frac{\partial p^{\alpha}}{\partial x^{\nu}}$ are related by the Hamilton-De DonderWeyl equations (the third one). From these expressions we recover the Euler-Lagrange $m$-vector fields and connections which are the solutions to the Lagrangian problem, and the HamiltonDe Donder-Weyl $m$-vector fields and connections which are the solutions to the Hamiltonian problem obtained in the above paragraph.

\section{Discussion and outlook}

Multisymplectic geometry and its application to describe classical field theories have been fields of increasing interest in the last years. A lot of well-known results in the realm of symplectic geometry and symplectic mechanics have been generalized also for the multisymplectic case, but there are many other problems which remain open. Next we review some of these results and problems, and their current status.

A fundamental result in symplectic geometry is the Darboux theorem. The analogous result also holds in some particular cases of multisymplectic forms (for instance, for volume forms). Nevertheless, in the general case, a multisymplectic manifold does not admit a system of Darboux coordinates for the multisymplectic form. In fact this is a problem arising from linear algebra: the classification of skew-symmetric tensors of degree greater than two is still an open problem. The kind of multisymplectic manifolds admitting Darboux coordinates has been identified [73], and they are those being locally multisymplectomorphic to bundles of forms (see also [33] for another approach to this problem).

Another interesting subject concerns to the definition of Poisson brackets in multisymplectic manifolds. This is a relevant point, for instance, for the further quantization of classical field theories. This problem has been studied in the realm of polysymplectic manifolds $[50,51]$ and for the multisymplectic case some recent contributions are [34, 35, 36]. However, the problem is not completely solved satisfactorily, and the research on this topic is still open.

In the same way, approaches for generalizing symplectic integrators to this geometric framework (i.e., the so-called multisymplectic integrators) have been studied in recent years, and numerical methods have been developed for solving the field equations, which are based on the use of these multisymplectic integrators [77, 79]. Research on this topic is in progress.

Another field of increasing interest in the last years is the study of systems in classical field theories with nonholonomic constraints. This is a meeting topic between honholonomic mechanics and classical field theories. The construction of the Lagrangian and Hamiltonian formalism, as well as other problems such as the study of symmetries and reduction have been analyzed for the $k$-symplectic formulation [72] and for the multisymplectic models in several works [10, 95, 96, 97, 98].

Further developments have not been achieved. For instance, the generalization of the Marsden-Weinstein reduction theorem [80] to the multisymplectic framework. Concerning reduction theory in general, only partial results about reduction by foliations are currently being studied [49]. The corresponding reduction theorem has been stated and proved for the $k$-symplectic formulation [84], but the theory of reduction of multisymplectic Lagrangian and Hamiltonian systems under the action of groups of symmetries is still under research, and only partial results have been achieved [17, 18, 19, 81].

The problem of quantization of classical field theories is another relevant topic to be developed. There are several works due to Kanatchikov devoted to geometric (pre)quantization of polysymplectic field theories $[53,54,55,56,57,58,59]$, some attempts for the $k$-symplectic case $[12,89]$, and other different approaches for the quantization of fields, in general (see, for instance, $[8,90])$. Nevertheless, the study of the geometric structures and obstructions to 
perform the geometric quantization program for covariant multisymplectic field theories is open to further research.

As a final remark, many of the subjects that we have presented in this work have been studied also for higher-order field theories (see, for instance, $[1,2,31,32,38,62,63,92,93]$ ). One of the problems of the first multisymplectic models for these theories was that the definition of the corresponding multisymplectic structure (the Poincaré-Cartan form) was ambiguous. This trouble have been solved recently [13]. But, in general, the problem of stating complete and satisfactory geometrical models for the Lagrangian and Hamiltonian formalisms of these kinds of theories, as well as other related topics (symmetries, constraint algorithms for the singular cases, quantization, ... ) are under development.

One can expect to see more work on all these subjects in the future.

\section{A Appendix}

\section{A.1 Multisymplectic manifolds}

Definition 7. Let $\mathcal{M}$ be a differentiable manifold, and $\Omega \in \Omega^{k}(\mathcal{M})(1<k \leq \operatorname{dim} \mathcal{M})$.

$\Omega$ is a multisymplectic form, and then $(\mathcal{M}, \Omega)$ is a multisymplectic manifold, if

1. $\Omega \in Z^{k}(\mathcal{M})$ (it is closed).

2. $\Omega$ is 1-nondegenerate; that is, for every $p \in \mathcal{M}$ and $X_{p} \in \mathrm{T}_{p} \mathcal{M}, i\left(X_{p}\right) \Omega_{p}=0 \Leftrightarrow X_{p}=0$.

If $\Omega$ is closed and 1-degenerate then it is a pre-multisymplectic form, and $(\mathcal{M}, \Omega)$ is a premultisymplectic manifold.

Multisymplectic manifolds of degree $k=2$ are the usual symplectic manifolds, and manifolds with a distinguished volume form are multisymplectic manifolds of degree its dimension. Other examples of multisymplectic manifolds are provided by compact semisimple Lie groups equipped with the canonical cohomology 3-class, symplectic 6-dimensional Calabi-Yau manifolds with the canonical 3-class, etc. There are no multisymplectic manifolds of degrees 1 or $\operatorname{dim} \mathcal{M}-1$ because $\operatorname{ker} \Omega$ is nonvanishing in both cases.

Another very important kind of multisymplectic manifold is the multicotangent bundle of a manifold $Q, \Lambda^{k}\left(\mathrm{~T}^{*} Q\right)$, that is, the bundle of $k$-forms in $Q$. This bundle is endowed with a canonical $k$-form $\Theta \in \Omega^{k}\left(\Lambda^{k}\left(\mathrm{~T}^{*} Q\right)\right.$, and then $\Omega:=-\mathrm{d} \Theta \in \Omega^{k+1}\left(\Lambda^{k}\left(\mathrm{~T}^{*} Q\right)\right.$ is a 1-nondegenerate form. Then the couple $\left(\Lambda^{k}\left(\mathrm{~T}^{*} Q\right), \Omega\right)$ is a multisymplectic manifold.

A local classification of multisymplectic forms can be done only for particular cases $[73,33]$.

\section{A.2 Multivector fields}

See [27] for details. Let $\mathcal{M}$ be a $n$-dimensional differentiable manifold. Sections of $\Lambda^{m}(\mathrm{TM})$ are called $m$-multivector fields in $\mathcal{M}$ (they are the contravariant skew-symmetric tensors of order $m$ in $\mathcal{M})$. We denote by $\mathfrak{X}^{m}(\mathcal{M})$ the set of $m$-multivector fields in $\mathcal{M}$. Then, $\mathcal{X} \in \mathfrak{X}^{m}(\mathcal{M})$ is locally decomposable if, for every $p \in \mathcal{M}$, there is an open neighbourhood $U_{p} \subset \mathcal{M}$ and $X_{1}, \ldots, X_{m} \in \mathfrak{X}\left(U_{p}\right)$ such that $\left.\mathcal{X}\right|_{U_{p}}=X_{1} \wedge \ldots \wedge X_{m}$.

A non-vanishing $\mathcal{X} \in \mathfrak{X}^{m}(\mathcal{M})$ and a $m$-dimensional distribution $\mathcal{D} \subset \mathrm{TM}$ are locally associated if there exists a connected open set $U \subseteq \mathcal{M}$ such that $\left.\mathcal{X}\right|_{U}$ is a section of $\left.\Lambda^{m} \mathcal{D}\right|_{U}$. If $\mathcal{X}, \mathcal{X}^{\prime} \in \mathfrak{X}^{m}(\mathcal{M})$ are non-vanishing multivector fields locally associated with the same distribution $\mathcal{D}$, on the same connected open set $U$, then there exists a non-vanishing function $f \in \mathrm{C}^{\infty}(U)$ such that $\left.\mathcal{X}^{\prime}\right|_{U}=f \mathcal{X}$. This fact defines an equivalence relation in the set of nonvanishing $m$-multivector fields in $\mathcal{M}$, whose equivalence classes will be denoted by $\{\mathcal{X}\}_{U}$. Then there is a one-to-one correspondence between the $m$-dimensional orientable distributions $\mathcal{D}$ in 
TM and the equivalence classes $\{\mathcal{X}\}_{\mathcal{M}}$ of non-vanishing, locally decomposable $m$-multivector fields in $\mathcal{M}$.

A non-vanishing, locally decomposable multivector field $\mathcal{X} \in \mathfrak{X}^{m}(\mathcal{M})$ is said to be integrable (resp. involutive) if its associated distribution is integrable (resp. involutive). If $\mathcal{X} \in \mathfrak{X}^{m}(\mathcal{M})$ is integrable (resp. involutive), then so is every other in its equivalence class $\{\mathcal{X}\}$, and all of them have the same integral manifolds. Moreover, Frobenius theorem allows us to say that a non-vanishing and locally decomposable multivector field is integrable if, and only if, it is involutive.

If $\pi: \mathcal{M} \rightarrow M$ is a fiber bundle, we are interested in the case where the integral manifolds of integrable multivector fields in $\mathcal{M}$ are sections of $\pi$. Thus, $\mathcal{X} \in \mathfrak{X}^{m}(\mathcal{M})$ is said to be $\pi$ transverse if, at every point $y \in \mathcal{M},\left(i(\mathcal{X})\left(\pi^{*} \beta\right)\right)_{y} \neq 0$, for every $\beta \in \Omega^{m}(M)$ with $\omega(\pi(y)) \neq 0$. Then, if $\mathcal{X} \in \mathfrak{X}^{m}(\mathcal{M})$ is integrable, it is $\pi$-transverse if, and only if, its integral manifolds are local sections of $\pi: \mathcal{M} \rightarrow M$. Finally, it is clear that classes of locally decomposable and $\pi$ transverse multivector fields $\{\mathcal{X}\} \subseteq \mathfrak{X}^{m}(\mathcal{M})$ are in one-to-one correspondence with orientable Ehresmann connection forms $\nabla$ in $\pi: \mathcal{M} \rightarrow M$. This correspondence is characterized by the fact that the horizontal subbundle associated with $\nabla$ is the distribution associated with $\{\mathcal{X}\}$. In this correspondence, classes of integrable locally decomposable and $\pi$-transverse $m$ multivector fields correspond to flat orientable Ehresmann connections.

\section{Acknowledgements}

I acknowledge the financial support of Ministerio de Educación y Ciencia, projects MTM 200504947, MTM 2008-00689/MTM and MTM 2008-03606-E/MTM. I wish to thank to Professors Miguel C. Muñoz-Lecanda and Xavier Gràcia for their comments, and to the referees, whose suggestions have allowed me to improve this work. Finally, thanks also to Mr. Jeff Palmer for his assistance in preparing the English version of the manuscript.

\section{References}

[1] Aldaya V., de Azcárraga J.A., Variational principles on $r$ th order jets of fibre bundles in field theory, J. Math. Phys. 19 (1978), 1869-1875.

[2] Aldaya V., de Azcárraga J.A., Higher-order Hamiltonian formalism in field theory, J. Phys. A: Math. Gen. 13 (1980), 2545-2551.

[3] Aldaya V., de Azcárraga J.A., Geometric formulation of classical mechanics and field theory, Riv. Nuovo Cimento 3 (1980), no. 10, 1-66.

[4] Awane A., k-symplectic structures, J. Math. Phys. 32 (1992), 4046-4052.

[5] Awane A., G-espaces k-symplectiques homogènes, J. Geom. Phys. 13 (1994), 139-157.

[6] Awane A., Goze M., Pfaffian systems, k-symplectic systems, Kluwer Academic Publishers, Dordrecht, 2000.

[7] Barbero-Liñán M., Echeverría-Enríquez A., Martín de Diego D., Muñoz-Lecanda M.C., Román-Roy N., Unified formalism for nonautonomous mechanical systems, J. Math. Phys. 49 (2008), 062902, 14 pages, arXiv:0803.4085.

[8] Bashkirov D., BV quantization of covariant (polysymplectic) Hamiltonian field theory, Int. J. Geom. Methods Mod. Phys. 1 (2004), 233-252, hep-th/0403263.

[9] Betounes D.E., Extension of the classical Cartan-form, Phys. Rev. D 29 (1984), 599-606.

[10] Binz E., de León M., Martín de Diego D., Socolescu D., Nonholonomic constraints in classical field theories, Rep. Math. Phys. 49 (2002), 151-166, math-ph/0201038.

[11] Binz E., Sniatycki J., Fisher H., Geometry of classical fields, North-Holland Mathematics Studies, Vol. 154, North-Holland Publishing Co., Amsterdam, 1988.

[12] Blaga A.M., The prequantization of $T_{k}^{1} \mathbb{R}^{n}$, in Differential Geometry and Its Applications, Proc. Conf. in honour Leonard Euler (Olomouc, 2007), World Sci. Publ., Hackensack, NJ, 2008, 217-222. 
[13] Campos C.M., de León M., Martín de Diego D., Vankerschaver J., Unambiguous formalism for higher-order Lagrangian field theories, J. Phys. A Math. Theor., to appear, arXiv:0906.0389.

[14] Cantrijn F., Ibort L.A., de León M., Hamiltonian structures on multisymplectic manifolds, Rend. Sem. Mat. Univ. Politec. Torino 54 (1996), 225-236.

[15] Cantrijn F., Ibort L.A., de León M., On the geometry of multisymplectic manifolds, J. Austral. Math. Soc. Ser. A 66 (1999), 303-330.

[16] Cariñena J.F., Crampin M., Ibort L.A., On the multisymplectic formalism for first order field theories, Differential Geom. Appl. 1 (1991), 345-374.

[17] Castrillón-López M., García-Pérez P.L., Ratiu T.S., Euler-Poincaré reduction on principal bundles, Lett. Math. Phys. 58 (2001), 167-180.

[18] Castrillón-López M., Marsden J.E., Some remarks on Lagrangian and Poisson reduction for field theories, J. Geom. Phys. 48 (2003), 52-83.

[19] Castrillón-López M., Ratiu T.S., Shkoller S., Reduction in principal fiber bundles: covariant Euler-Poincaré equations, Proc. Amer. Math. Soc. 128 (2000), 2155-2164, math.DG/9908102.

[20] Cortés J., Martínez S., Cantrijn F., Skinner-Rusk approach to time-dependent mechanics, Phys. Lett. A 300 (2002), 250-258, math-ph/0203045.

[21] Dedecker P., On the generalization of symplectic geometry to multiple integrals in the calculus of variations, in Differential Geometrical Methods in Mathematical Physics (Proc. Sympos., Univ. Bonn, Bonn, 1975) Lecture Notes in Math., Vol. 570, Springer, Berlin, 1977, 395-456.

[22] Dedecker P., Problèmes variationnels dégénérés, C.R. Acad. Sci. Paris Sér. A-B 286 (1978), A547-A550.

[23] Echeverría-Enríquez A., de León M., Muñoz-Lecanda M.C., Román-Roy N., Extended Hamiltonian systems in multisymplectic field theories, J. Math. Phys. 48 (2007), 112901, 30 pages, math-ph/0506003.

[24] Echeverría-Enríquez A., López C., Marín-Solano J., Muñoz-Lecanda M.C., Román-Roy N., LagrangianHamiltonian unified formalism for field theory, J. Math. Phys. 45 (2004), 360-380, math-ph/0212002.

[25] Echeverría-Enríquez A., Muñoz-Lecanda M.C., Variational calculus in several variables: a Hamiltonian approach, Ann. Inst. Henri Poincaré Phys. Theór. 56 (1992), 27-47.

[26] Echeverría-Enríquez A., Muñoz-Lecanda M.C., Román-Roy N., Geometry of Lagrangian first-order classical field theories, Fortschr. Phys. 44 (1996), 235-280, dg-ga/9505004.

[27] Echeverría-Enríquez A., Muñoz-Lecanda M.C., Román-Roy N., Multivector fields and connections: setting Lagrangian equations in field theories, J. Math. Phys. 39 (1998), 4578-4603, dg-ga/9707001.

[28] Echeverría-Enríquez A., Muñoz-Lecanda M.C., Román-Roy N., Multivector field formulation of Hamiltonian field theories: equations and symmetries, J. Phys. A: Math. Gen. 32 (1999), 8461-8484, math-ph/9907007.

[29] Echeverría-Enríquez A., Muñoz-Lecanda M.C., Román-Roy N., On the Multimomentum bundles and the Legendre Maps in field theories, Rep. Math. Phys. 45 (2000), 85-105, math-ph/9904007.

[30] Echeverría-Enríquez A., Muñoz-Lecanda M.C., Román-Roy N., Geometry of multisymplectic Hamiltonian first-order field theories, J. Math. Phys. 41 (2000), 7402-7444, math-ph/0004005.

[31] Ferraris M., Francaviglia M., Applications of the Poincaré-Cartan form in higher order field theories, in Differential Geometry and Its Applications (Brno, 1986), Math. Appl. (East European Ser.), Vol. 27, Reidel, Dordrecht, 1987, 31-52.

[32] Ferraris M., Francaviglia M., Intrinsic ADM formalism for generally covariant higher-order field theories, Atti Sem. Mat. Fis. Univ. Modena 37 (1989), 61-78.

[33] Forger M., Gomes L., Multisymplectic and polysymplectic structures on fiber bundles, arXiv:0708.1586.

[34] Forger M., Paufler C., Römer H., A general construction of Poisson brackets on exact multisymplectic manifolds, Rep. Math. Phys. 51 (2003), 187-195, math-ph/0208037.

[35] Forger M., Paufler C., Römer H., The Poisson bracket for Poisson forms in multisymplectic field theory, Rev. Math. Phys. 15 (2003), 705-743, math-ph/0202043.

[36] Forger M., Paufler C., Römer H., Hamiltonian multivector fields and Poisson forms in multisymplectic field theory, J. Math. Phys. 46 (2005), 112903, 29 pages, math-ph/0407057.

[37] García P.L., The Poincaré-Cartan invariant in the calculus of variations, Symposia Mathematica, Vol. 14 (Convegno di Geometria Simplettica e Fisica Matematica, INDAM, Rome, 1973), Academic Press, London, 1974, 219-246. 
[38] García P.L., Muñoz J., On the geometrical structure of higher order variational calculus, Proceedings of the IUTAM-ISIMM symposium on modern developments in analytical mechanics, Vol. I (Torino, 1982), Editors M. Francaviglia and A. Lichnerowicz, Atti Accad. Sci. Torino Cl. Sci. Fis. Mat. Natur. 117 (1983), suppl. 1, $127-147$.

[39] Giachetta G., Mangiarotti L., Sardanashvily G., New Lagrangian and Hamiltonian methods in field theory, World Scientific Publishing Co., Inc., River Edge, NJ, 1997.

[40] Goldschmidt H., Sternberg S., The Hamilton-Cartan formalism in the calculus of variations, Ann. Inst. Fourier (Grenoble) 23 (1973), 203-267.

[41] Gotay M.J., A multisymplectic framework for classical field theory and the calculus of variations. I. Covariant Hamiltonian formalism, in Mechanics, Analysis and Geometry: 200 Years after Lagrange, Editor M. Francaviglia, North-Holland, Amsterdam, 1991, 203-235.

[42] Gotay M.J., A multisymplectic framework for classical field theory and the calculus of variations. II. Space + time decomposition, Differential Geom. Appl. 1 (1991), 375-390.

[43] Gotay M.J., Isenberg J., Marsden J.E., Momentum maps and classical relativistic fields. I. Covariant theory, MSRI Preprints, 1999.

[44] Gotay M.J., Isenberg J., Marsden J.E., Momentum maps and classical relativistic fields. II. Canonical analysis of field theories, MSRI Preprints, 1999.

[45] Gràcia X., Martín R., Geometric aspects of time-dependent singular differential equations, Int. J. Geom. Methods Mod. Phys. 2 (2005), 597-618.

[46] Günther C., The polysymplectic Hamiltonian formalism in the field theory and the calculus of variations. I. The local case, J. Differential Geom. 25 (1987), 23-53.

[47] Hélein F., Kouneiher J., Finite dimensional Hamiltonian formalism for gauge and quantum field theories, J. Math. Phys. 43 (2002), 2306-2347, math-ph/0004020.

[48] Hélein F., Kouneiher J., Covariant Hamiltonian formalism for the calculus of variations with several variables: Lepage-Dedecker versus De Donder-Weyl, Adv. Theor. Math. Phys. 8 (2004), 565-601, math-ph/0401046.

[49] Ibort L.A., Multisymplectic manifolds: general aspects and particular situations, Proceedings of the IX Fall Workshop on Geometry and Physics (Vilanova i la Geltrú, 2000), Editors X. Gràcia, J. Marín-Solano, M.C. Muñoz-Lecanda and N. Román-Roy, Publ. R. Soc. Mat. Esp., Vol. 3, R. Soc. Mat. Esp., Madrid, 2001, 79-88.

[50] Kanatchikov I.V., From the Poincaré-Cartan form to a Gerstenhaber algebra of the Poisson brackets in field theory, in Quantization, coherent states, and complex structures (Bialowieza, 1994), Plenum Press, New York, 1995, 173-183, hep-th/9511039.

[51] Kanatchikov I.V., On field-theoretic generalizations of a Poisson algebra, Rep. Math. Phys. 40 (1997), 225-234, hep-th/9710069.

[52] Kanatchikov I.V., Canonical structure of classical field theory in the polymomentum phase space, Rep. Math. Phys. 41 (1998), 49-90, hep-th/9709229.

[53] Kanatchikov I.V., Toward the Born-Weyl quantization of fields, Internat. J. Theoret. Phys. 37 (1998), 333-342, quant-ph/9712058.

[54] Kanatchikov I.V., De Donder-Weyl theory and a hypercomplex extension of quantum mechanics to field theory, Rep. Math. Phys. 43 (1999), 157-170, hep-th/9810165.

[55] Kanatchikov I.V., Precanonical perspective in quantum gravity, Nuclear Phys. B Proc. Suppl. 88 (2000), 326-330, gr-qc/0004066.

[56] Kanatchikov I.V., Geometric (pre)quantization in the polysymplectic approach to field theory, in Differential geometry and its applications (Opava, 2001), Math. Publ., Vol. 3, Silesian Univ. Opava, Opava, 2001, 309321, hep-th/0112263.

[57] Kanatchikov I.V., Precanonical quantization and the Schrödinger wave functional, Phys. Lett. A 283 (2001), 25-36, hep-th/0012084.

[58] Kanatchikov I.V., Precanonical quantum gravity: quantization without the space-time decomposition, Internat. J. Theoret. Phys. 40 (2001), 1121-1149, gr-qc/0012074.

[59] Kanatchikov I.V., Precanonical quantization of Yang-Mills fields and the functional Schrödinger representation, Rep. Math. Phys. 53 (2004), 181-193, hep-th/0301001.

[60] Kijowski J., A finite-dimensional canonical formalism in the classical field theory, Comm. Math. Phys. 30 (1973), 99-128. 
[61] Kijowski J., Tulczyjew W.M., A symplectic framework for field theories, Lecture Notes in Physics, Vol. 170, Springer-Verlag, Berlin - New York, 1979.

[62] Kouranbaeva S., Shkoller S., A variational approach to second-order multisymplectic field theory, J. Geom. Phys. 35 (2000), 333-366, math.DG/9909100.

[63] Krupka D., On the higher order Hamilton theory in fibered spaces, in Proceedings of the Conference on Differential Geometry and Its Applications, Part 2, Univ. J. E. Purkyne, Brno, 1984, 167-183.

[64] Krupka D., Regular Lagrangians and Lepagean forms, in Differential Geometry and Its Applications (Brno, 1986), Math. Appl. (East European Ser.), Vol. 27, Reidel, Dordrecht, 1987, 111-148.

[65] Krupkova O., Hamiltonian field theory, J. Geom. Phys. 43 (2002), 93-132.

[66] Krupkova O., Smetanova D., On regularization of variational problems in first-order field theory, in Proceedings of the 20th Winter School "Geometry and Physics" (Srni, 2000), Rend. Circ. Mat. Palermo (2) Suppl. 66 (2001), 133-140.

[67] Krupkova O., Smetanova D., Legendre transformation for regularizable Lagrangians in field theory, Lett. Math. Phys. 58 (2002), 189-204, math-ph/0111004.

[68] Lawson J.K., A frame bundle generalization of multisymplectic geometries, Rep. Math. Phys. 45 (2000), 183-205, dg-ga/9706008.

[69] de León M., Marín-Solano J., Marrero J.C., A geometrical approach to classical field theories: a constraint algorithm for singular theories, in New Developments in Differential Geometry (Debrecen, 1994), Editors L. Tamassi and J. Szenthe, Math. Appl., Vol. 350, Kluwer Acad. Publ., Dordrecht, 1996, 291-312.

[70] de León M., Marín-Solano J., Marrero J.C., Muñoz-Lecanda M.C., Román-Roy N., Pre-multisymplectic constraint algorithm for field theories, Int. J. Geom. Methods Mod. Phys. 2 (2005), 839-871, math-ph/0506005.

[71] de León M., Marrero J.C., Martín de Diego D., A new geometrical setting for classical field theories, in Classical and Quantum Integrability (Warsaw, 2001), Banach Center Pub., Vol. 59, Inst. of Math., Polish Acad. Sci., Warsawa, 2003, 189-209, math-ph/0202012.

[72] de León M., Martín de Diego D., Salgado M., Vilariño S., $k$-symplectic formalism on Lie algebroids, J. Phys. A: Math. Theor. 42 (2009), 385209, 31 pages, arXiv:0905.4585.

[73] de León M., Martín de Diego D., Santamaría-Merino A., Tulczyjew's triples and Lagrangian submanifolds in classical field theories, in Applied Differential Geometry and Mechanics, Editors W. Sarlet and F. Cantrijn, Univ. of Gent, Gent, Academia Press, 2003, 21-47, math-ph/0302026.

[74] de León M., Martín de Diego D., Santamaría-Merino A., Symmetries in classical field theories, Int. J. Geom. Methods Mod. Phys. 1 (2004), 651-710, math-ph/0404013.

[75] de León M., Merino E., Oubiña J.A., Rodrigues P., Salgado M., Hamiltonian systems on $k$-cosymplectic manifolds, J. Math. Phys. 39 (1998), 876-893.

[76] de León M., Merino E., Salgado M., k-cosymplectic manifolds and Lagrangian field theories, J. Math. Phys. 42 (2001), 2092-2104.

[77] Marsden J.E., Patrick G.W., Shkoller S., Multisymplectic geometry, variational integrators, and nonlinear PDEs, Comm. Math. Phys. 199 (1998), 351-395, math.DG/9807080.

[78] Marsden J.E., Patrick G.W., Shkoller S., West M., Variational methods, multisymplectic geometry and continuum mechanics, J. Geom. Phys. 38 (2001), 253-284, math.DG/0005034.

[79] Marsden J.E., Shkoller S., Multisymplectic geometry, covariant Hamiltonians and water waves, Math. Proc. Cambridge Philos. Soc. 125 (1999), 553-575, math.DG/9807086.

[80] Marsden J.E., Weinstein A., Reduction of symplectic manifolds with symmetry, Rep. Math. Phys. 5 (1974), $121-130$.

[81] Marsden J.E., Weinstein A., Some comments on the history, theory, and applications of symplectic reduction, in Quantization of Singular Symplectic Quotiens, Editors N. Landsman, M. Pflaum and M. Schlichenmanier, Progr. Math., Vol. 198, Birkhäuser, Basel, 2001, 1-19.

[82] Martínez E., Classical field theory on Lie algebroids: multisymplectic formalism, math.DG/0411352.

[83] Martínez E., Classical field theories on Lie algebroids: variational aspects, J. Phys. A: Math. Gen. 38 (2005), 7145-7160, math-dg/0410551.

[84] Munteanu F., Rey A.M., Salgado M., The Günther's formalism in classical field theory: momentum map and reduction, J. Math. Phys. 45 (2004), 1730-1751.

[85] McLean M., Norris L.K., Covariant field theory on frame bundles of fibered manifolds, J. Math. Phys. 41 (2000), 6808-6823. 
[86] Norris L.K., Generalized symplectic geometry on the frame bundle of a manifold, in Differential Geometry: Geometry in Mathematical Physics and Related Topics (Los Angeles, CA, 1990), Proc. Sympos. Pure Math., Vol. 54, Part 2, Amer. Math. Soc., Providence, RI, 1993, 435-465.

[87] Norris L.K., n-symplectic algebra of observables in covariant Lagrangian field theory, J. Math. Phys. 42 (2001), 4827-4845.

[88] Paufler C., Römer H., Geometry of Hamiltonian $n$-vector fields in multisymplectic field theory, J. Geom. Phys. 44 (2002), 52-69, math-ph/0102008.

[89] Puta M., Chirici S., Merino E., On the prequantization of $\left(T_{k}^{1}\right)^{*} \mathbb{R}^{n}$, Bull. Math. Soc. Sci. Math. Roumanie (N.S.) 44(92) (2001), 277-284.

[90] Sardanashvily G., Multimomentum canonical quantization of fields, Hadronic J. 17 (1994), 227-245.

[91] Sardanashvily G., Generalized Hamiltonian formalism for field theory. Constraint systems, World Scientific Publishing Co., Inc., River Edge, NJ, 1995.

[92] Saunders D.J., The Geometry of jet bundles, London Mathematical Society Lecture Note Series, Vol. 142, Cambridge University Press, Cambridge, 1989.

[93] Saunders D.J., Crampin M., On the Legendre map in higher-order field theories, J. Phys. A: Math. Gen. 23 (1990), 3169-3182.

[94] Skinner R., Rusk R., Generalized Hamiltonian dynamics. I. Formulation on $T^{*} Q \oplus T Q$, J. Math. Phys. 24 (1983), 2589-2594.

[95] Vankerschaver J., Cantrijn F., de León M., Martín de Diego D., Geometric aspects of nonholonomic field theories, Rep. Math. Phys. 56 (2005), 387-411, math-ph/0506010.

[96] Vankerschaver J., The momentum map for nonholonomic field theories with symmetry, Int. J. Geom. Methods Mod. Phys. 2 (2005), 1029-1041, math-ph/0507059.

[97] Vankerschaver J., Euler-Poincaré reduction for discrete field theories, J. Math. Phys. 48 (2007), 032902, 17 pages, math-ph/0606033.

[98] Vankerschaver J., Martín de Diego D., Symmetry aspects of nonholonomic field theories, J. Phys. A: Math. Theor. 41 (2008), 035401, 17 pages, arXiv:0712.2272. 